\title{
Klimarelevanz von Ersatzbrennstoffen - Anwendung und Vergleich verschiedener Bestimmungsmethoden
}

\author{
Therese Schwarzböck · Helmut Rechberger · Philipp Aschenbrenner · Stefan Spacek · Sönke Szidat • \\ Johann Fellner
}

Online publiziert: 24. Januar 2018

(C) Der/die Autor(en) 2018. Dieser Artikel ist eine Open-Access-Publikation.

Zusammenfassung Der Einsatz von alternativen Brennstoffen - wie aus Abfall hergestellte Ersatzbrennstoffe (EBS) - kann in industriellen thermischen Verwertungsanlagen (beispielsweise in Zementwerken) neben der Einsparung von Primärrohstoffen auch zu einer Reduzierung der klimarelevanten $\mathrm{CO}_{2-}$ Emissionen führen. Um diese $\mathrm{CO}_{2}$-Einsparungen nachzuweisen, bedarf es einer Methode, die der Heterogenität der Abfallgemische gerecht wird und den fossilen Kohlenstoffanteil bzw. den fossilen $\mathrm{CO}_{2}$-Emissionsfaktor der EBS zuverlässig bestimmen lässt. Die Studie befasst sich mit der Erprobung einer alternativen Bestimmungsmethode, der sogenannten adaptierten Bilanzenmethode $(\mathrm{aBM})$, die auf der Bestimmung der Elementarzusammensetzung des EBS beruht. Insgesamt wurden sechs verschiedene EBS auf ihre Klimarelevanz mittels aBM untersucht und mit Ergebnissen standardisierter Verfahren verglichen. Dabei zeigte sich eine sehr gute Übereinstimmung der aBM-Werte mit denen der Radiokarbonmethode $\left({ }^{14} \mathrm{C}\right.$-Methode), die als Vergleichsmethode herangezogen werden kann. Die mittlere Abweichung von der ${ }^{14} \mathrm{C}-\mathrm{Me}$ thode lag bei $0,6 \pm 1,4 \%$ absolut bezogen auf den mittleren fossilen Kohlenstoffanteil. Für die zwei weiteren standardisierten Methoden (Selektive Lösemethode und Manuelle Sor-

T. Schwarzböck, MSc $(\bowtie)$.

Univ.-Prof. DI Dr. H. Rechberger .

P. Aschenbrenner · S. Spacek, MSc .

Ass.-Prof. DI Dr. J. Fellner

Institut für Wassergüte und

Ressourcenmanagement, Technische

Universität Wien, Karlsplatz 13,

E226/2, 1040 Wien, Österreich

therese.schwarzboeck@tuwien.ac.at

Ass.-Prof. Dr. S. Szidat

Labor zur Analyse von

Radiokohlenstoff mit AMS

(LARA), Universität Bern,

Freiestraße 3, 3012 Bern, Schweiz tierung) zeigten sich beim Vergleich der Ergebnisse deutliche methodische Einschränkungen in Abhängigkeit von der Zusammensetzung der EBS. Die $\mathrm{aBM}$ ist damit neben der analytisch aufwendigen ${ }^{14} \mathrm{C}$-Methode das einzige Bestimmungsverfahren, das unabhängig vom EBS-Typ zuverlässige Werte zur Klimarelevanz generiert. Zudem weist die Praktikabilität (Zeit- und Kostenaufwand) der aBM, insbesondere bei Routineanwendungen, Vorteile gegenüber standardisierten Verfahren auf. Einzig bei erstmaliger Anwendung ist eine Ermittlung von EBS-spezifischen Eingangswerten zur Elementarzusammensetzung der enthaltenen biogenen und fossilen Materialien notwendig, welche mit erhöhtem Aufwand verbunden sein kann.

\section{Climate-relevance of refuse- derived fuels - Application and comparison of different determination methods}

Abstract Refuse-derived fuels (RDF) are utilized in industrial processes (e.g. cement plants) to reduce costs for primary energy carriers, to lessen natural resource consumption, but also to lower the amount of climate-relevant $\mathrm{CO}_{2}$ emissions associated with the production process. In order to account for the $\mathrm{CO}_{2}$ savings, a practical method is required which accounts for the heterogeneity of RDF and allows the share of fossil carbon and fossil $\mathrm{CO}_{2}$ emissions to be reliably determined. The study examines an alternative method, the so-called adapted Balance Method $(\mathrm{aBM})$, which relies on the determination of the elementary composition of the RDF. Six different RDFs were investigated by means of aBM and the results were compared to three standardized methods. The results of aBM are in excellent agreement with the ones of ${ }^{14} \mathrm{C}$-Method which is regarded as reference method (mean deviation of $0.6 \pm 1.4 \%$ absolute based on the share of fossil carbon). Both other standardized methods, the Selective Dissolution Method and the method of Manual Sorting show methodological limitations leading to allegedly false estimates for single RDFs. Thus, the aBM is-besides the ${ }^{14} \mathrm{C}$-Method, which requires highly specialist equipment and personnel-the only determination method which delivers reliable data on the climate-relevant $\mathrm{CO} 2$ emissions independently of the type of RDF. Further benefits of the aBM in comparison to the standardized methods include its practicability for routine application (expenditure of time and money). Only during initial application of the aBM the determination of RDF-specific input parameters (elemental composition of biogenic and fossil materials in the RDF) may lead to increased efforts.

\section{Einleitung}

Der Einsatz von Ersatzbrennstoffen in energieintensiven Industriebranchen, wie beispielsweise in Zementwerken oder in der Stahlproduktion, hat in den letzten Jahren stark an Bedeutung gewonnen. Vor dem Hintergrund der Einsparung von Primärrohstoffen und der Reduktion von klimarelevanten $\mathrm{CO}_{2}$-Emissionen, liegt beispielsweise die thermische Substitutionsrate in der Zementindustrie in der Europäischen Union bei mittlerweile mehr als 34 \% (VÖZ 2015). In einzelnen Ländern werden bereits mehr als $50 \%$ des thermischen Energiebedarfs in Zementwerken durch Ersatzbrennstoffe gedeckt z.B. Österreich $76 \%$, Deutschland $65 \%$, Belgien $60 \%$, Schweiz $54 \%$ (cemsuisse 2016; Rahman et al. 2015; VDZ 2017; VÖZ 2015).

Die Untersuchung und Deklaration von technischen und ökologischen Eigenschaften alternativer Brennstoffe ist sowohl für Produzenten als auch für deren Abnehmer von Relevanz. Beispielsweise ist laut europäischem Standard EN 15359:2011 neben der 
Hier steht eine Anzeige.

Springer 
Hier steht eine Anzeige.

Springer 
Tab. 1 Gegenüberstellung der Methoden zur Bestimmung des fossilen/biogenen Anteils in Abfallgemischen und Ersatzbrennstoffen

\begin{tabular}{|c|c|c|c|c|c|}
\hline & $\begin{array}{l}\text { MS } \\
\text { (a) }\end{array}$ & $\int^{\text {SLM }}$ & ${ }^{14} \mathrm{Cr}$ & 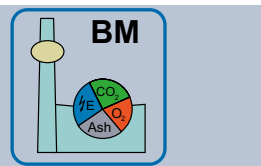 & $\begin{array}{|lll|} & \mathrm{aBM} \\
\mathrm{N} & \mathrm{C} \\
& \mathrm{S} & \mathrm{O} \\
\end{array}$ \\
\hline & Manuelle Sortierung & Selektive Lösemethode & Radiokarbonmethode & Bilanzenmethode & Adaptierte Bilanzenmethode \\
\hline Standardisiert & $\begin{array}{l}\boldsymbol{v} \\
\text { EN 15440:2011 }\end{array}$ & $\begin{array}{l}\boldsymbol{V} \\
\text { EN 15440:2011 }\end{array}$ & $\begin{array}{l}\boldsymbol{v} \\
\text { EN 15440:2011 }\end{array}$ & $\begin{array}{l}\boldsymbol{V} \\
\text { ISO 18466:2016 }\end{array}$ & - \\
\hline Grundprinzip & $\begin{array}{l}\text { Manuelle Sortierung in } \\
\text { biogen und fossil }\end{array}$ & $\begin{array}{l}\text { Chemische Lösung von } \\
\text { biogenen Massenantei- } \\
\text { len }\end{array}$ & $\begin{array}{l}\text { Unterschiedlicher Ge- } \\
\text { halt des }{ }^{14} \mathrm{C} \text {-Isotops in } \\
\text { biogenen und fossilen } \\
\text { Anteilen }\end{array}$ & $\begin{array}{l}\text { Abgleich von Massen-, } \\
\text { Stoff- und Energiebi- } \\
\text { lanzgleichungen in } \\
\text { MVA }\end{array}$ & $\begin{array}{l}\text { Abgleich von Stoffbilanzglei- } \\
\text { chungen }(C, H, N, S, 0)\end{array}$ \\
\hline Analyse von & Feststoffprobe & Feststoffprobe & $\begin{array}{l}\text { Feststoffprobe oder } \\
\text { Rauchgas }\end{array}$ & Anlagenbetriebsdaten & Feststoffprobe \\
\hline \multicolumn{6}{|c|}{ Bestimmung von fossil/biogen: } \\
\hline Massenanteil & $\checkmark$ & $\checkmark$ & - & $\checkmark$ & $\checkmark$ \\
\hline $\begin{array}{l}\text { Kohlenstoffanteil } \\
\left.\text { ( } \mathrm{CO}_{2} \text {-Emissionen }\right)\end{array}$ & $(\boldsymbol{\sim})$ & $(\boldsymbol{\sim})$ & $\checkmark$ & $\checkmark$ & $\checkmark$ \\
\hline Energieanteil & $(\boldsymbol{\sim})$ & $(\mathscr{})$ & $(\boldsymbol{\sim})$ & $\checkmark$ & $\checkmark$ \\
\hline
\end{tabular}

verpflichtenden Deklaration des Heizwertes, des Chlor- und Quecksilbergehalts auch der Biomassegehalt optional auszuweisen. Als Biomasse in Ersatzbrennstoffen ist derjenige Anteil zu verstehen, der biogenen Ursprungs ist (Papier, Holz, Gartenabfälle, etc.) und daher als erneuerbare Energiequelle gilt. Demnach sind die bei der thermischen Verwertung entstehenden $\mathrm{CO}_{2}$-Emissionen als nicht klimarelevant einzustufen. Dadurch ergibt sich durch den Einsatz von biomassehaltigen Ersatzbrennstoffen eine Einsparung klimarelevanter $\mathrm{CO}_{2}$-Emissionen im Vergleich zum Einsatz von fossilen Brennstoffen (wie Kohle, Erdöl, Erdgas). Um die tatsächliche Einsparung zu bestimmen und entsprechend nachzuweisen (z.B. im Zuge des Erwerbs von Emissionszertifikaten), ist es erforderlich, den fossilen Kohlenstoffanteil bzw. den fossilen $\mathrm{CO}_{2}$-Emissionsfaktor der eingesetzten Ersatzbrennstoffe zu bestimmen. Durch die typischerweise stark variierende Zusammensetzung der Ausgangsmaterialien für die Produktion von Ersatzbrennstoffen (wie Siedlungsabfälle, Gewerbeabfälle), ist eine zuverlässige Aussage jedoch nur bei periodisch wiederkehrender $\mathrm{Be}$ stimmung des fossilen Anteils gegeben. Generell bedarf es einer Bestimmungsmethode, die der Heterogenität der Abfallgemische gerecht wird, verlässliche Werte liefert und mit vertretbarem finanziellem und zeitlichem Aufwand verbunden ist.

\section{Verfügbare Methoden zur} Bestimmung der Klimarelevanz von Ersatzbrennstoffen

Bisher werden drei Methoden zur Bestimmung des fossilen Anteils in Ersatzbrennstoffen in der Norm EN 15440:2011 angeführt: Manuelle Sortierung (MS), Selektive Lösemethode (SLM) und Radiokarbonmethode $\left({ }^{14} \mathrm{C}\right.$ Methode). Alle drei Methoden sind auf feste Ersatzbrennstoffproben (sprich vor der thermischen Verwertung) anwendbar, jedoch teils limitiert in ihrer Anwendbarkeit und Parameterbereitstellung (Tab. 1). Eine kostengünstige Alternative stellt die sogenannte Bilanzenmethode (BM) dar, die kürzlich im Standard ISO 18466:2016 beschrieben wurde und in ihrer ursprünglichen Version eine Auswertung von Betriebsdaten von Müllverbrennungsanlagen (MVA) vorsieht (sprich nach der thermischen Verwertung durch Messungen im Rauchgas bzw. anderer Anlagenbetriebsdaten). Basierend auf dieser Methode wurde die adaptierte Bilanzenmethode (aBM) entwickelt, welche nach dem gleichen Grundprinzip wie die Ursprungsversion arbeitet (Abgleich von Bilanzgleichungen), jedoch eine Charakterisierung von Feststoffproben im Labor erlaubt. Damit können diese neue Methode sowie die bereits standardisierten Methoden MS, SLM, und ${ }^{14} \mathrm{C}$-Methode auf Ersatzbrennstoffe vor deren thermischer Verwertung angewandt werden. Bisherige Untersu- chungen zeigen, dass die aBM einige methodische Vorteile bietet und bei Anwendung auf vordefinierte Abfallgemische präzise und richtige Ergebnisse liefert (Aschenbrenner et al. 2009; Fellner et al. 2011; Schwarzböck et al. 2016) Beispielsweise können diverse Parameter (wie der fossile/biogene MassenKohlenstoff- und Energieanteil, Emissionsfaktoren, Kunststoffgehalt) direkt abgeleitet werden ohne Zusatzinformationen oder -analysen zu benötigen, wie dies bei den standardisierten Methoden der Fall ist (Tab. 1). Zudem konnten im Vergleich zu alternativen Bestimmungsmethoden verringerte Unsicherheiten beobachtet werden (Schwarzböck et al. 2016).

\section{Zielsetzung der Studie}

Übergeordnetes Ziel der vorliegenden Studie ist es, die adaptierte Bilanzenmethode (aBM) an verschiedenen Ersatzbrennstoffproben zu testen und mit standardisierten Verfahren zu vergleichen. Letzteres ist als Validierung der aBM zu verstehen.

Im Konkreten werden im vorliegenden Beitrag Untersuchungen mit realen Ersatzbrennstoffen vorgestellt und Fragen zur Zuverlässigkeit, Robustheit sowie praktischen Anwendbarkeit der neuen Methode behandelt. Dazu wurden

- Sortierungen durchgeführt um EBSspezifische Eingangsparameter der 
Tab. 2 Untersuchte Ersatzbrennstoffe und angewandte Methoden

\begin{tabular}{|c|c|c|c|c|c|c|}
\hline & Herkunft & $\begin{array}{l}\text { Anzahl an Proben } \\
\text { (Tagesstichproben) }\end{array}$ & MS & SLM & ${ }^{14} \mathrm{C}$ & $\mathrm{aBM}$ \\
\hline Papier Rejekt & Ausgeschleustes Material aus der Altpapieraufbereitung & $\begin{array}{l}15 \text { (je rund } 10 \mathrm{~kg} \\
\text { trocken) }\end{array}$ & $\checkmark$ & $\checkmark$ & $\boldsymbol{v}$ & $\checkmark$ \\
\hline EBS 1 & $\begin{array}{l}\text { EBS produziert aus voraufbereiteten Siedlungsabfällen sowie } \\
\text { Gewerbe- und Industrieabfällen }\end{array}$ & 8 (je rund $5 \mathrm{~kg}$ trocken) & $\boldsymbol{V}$ & $\checkmark$ & $\checkmark$ & $\boldsymbol{\nu}$ \\
\hline EBS 2 & EBS produziert aus vorwiegend Gewerbe- und Industrieabfällen & 8 (je rund $5 \mathrm{~kg}$ trocken) & $\boldsymbol{v}$ & $\boldsymbol{\nu}$ & $\boldsymbol{v}$ & $\boldsymbol{v}$ \\
\hline EBS 3 & EBS produziert aus vorwiegend Gewerbe- und Industrieabfällen & 3 & $\checkmark$ & - & - & $\checkmark$ \\
\hline EBS 4 & EBS produziert aus vorwiegend Gewerbe- und Industrieabfällen & 2 & $\checkmark$ & - & - & $\checkmark$ \\
\hline EBS 5 & EBS produziert aus vorwiegend Gewerbe- und Industrieabfällen & 3 & $\boldsymbol{\nu}$ & - & - & $\boldsymbol{\nu}$ \\
\hline
\end{tabular}

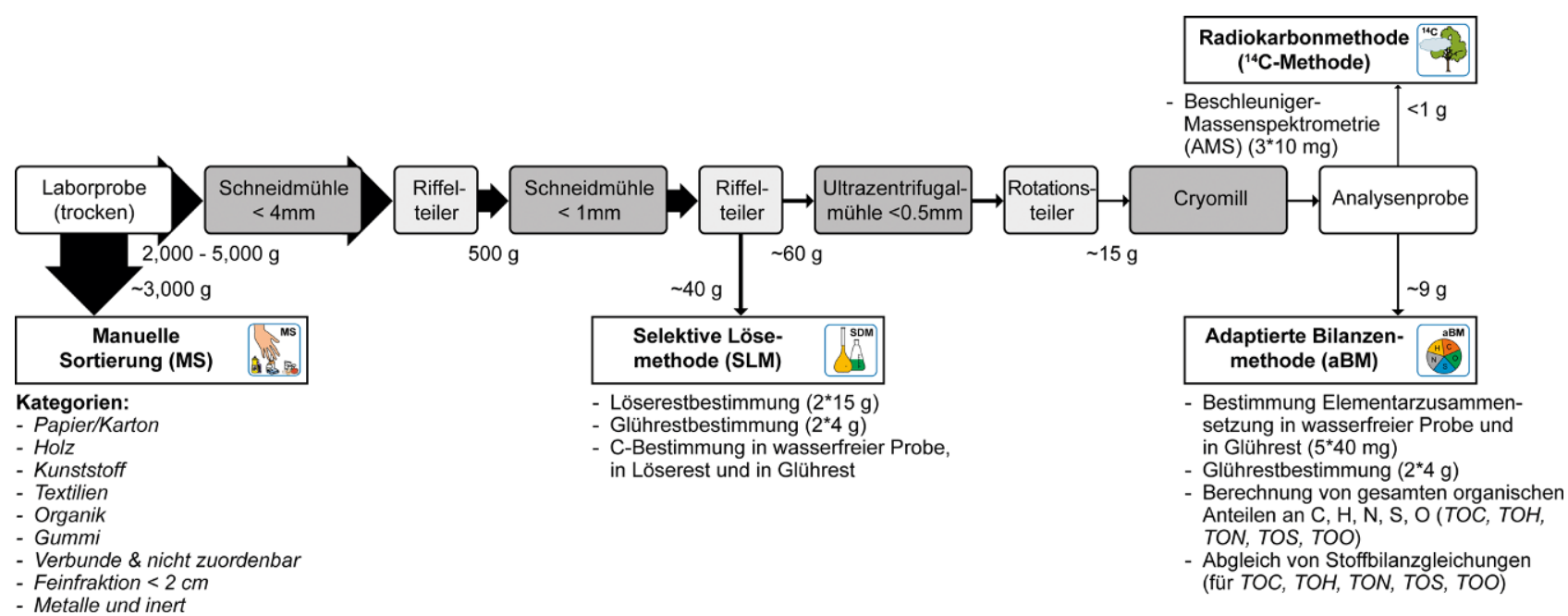

Abb. 1 Schema der Probenaufbereitung und der angewandten Bestimmungsmethoden

$\mathrm{aBM}$ zu generieren und mit Literaturdaten zu vergleichen,

- Ergebnisse aus der Anwendung der aBM mit Ergebnissen der drei bereits standardisierten Methoden verglichen und

- die Praktikabilität (Arbeitsaufwand, Verfügbarkeit, Kosten) der verfügbaren Bestimmungsmethoden gegenübergestellt.

\section{Methodik}

\subsection{Untersuchte Ersatzbrennstoffe und Probenaufbereitung}

Im Zuge der Studie wurden Proben sechs verschiedener Ersatzbrennstoffe mithilfe der aBM auf ihre Klimarelevanz untersucht (Tab. 2), wobei für alle Ersatzbrennstoffe auch eine Manuelle Sortierung (MS) durchgeführt wurde. Zusätzlich wurden für drei der EBS die Selektive Lösemethode (SLM) sowie die Radiokarbonmethode $\quad\left({ }^{14} \mathrm{C}\right.$-Methode $)$ (Tab. 2) angewandt. Die Methoden werden in Abschn. 4.2-4.5 näher erläutert.
In Abhängigkeit von der jeweiligen Analysemethode sind verschiedene Aufbereitungsschritte für die EBSProben notwendig (Abb. 1). Die aBM erfordert beispielsweise eine sehr feine Partikelgröße $(0,5 \mathrm{~mm}$ oder kleiner), da die Elementaranalyse mittels katalytischer Verbrennung nur eine geringe Probeneinwaage (rund $40 \mathrm{mg}$ pro Analyse) zulässt. Dies ist ähnlich bei der ${ }^{14} \mathrm{C}$-Methode. Die SLM hingegen sieht die chemische Behandlung der Proben bei einer Partikelgröße von $<1 \mathrm{~mm}$ vor.

\subsection{Adaptierte Bilanzenmethode (aBM)}

\subsubsection{Methodenbeschreibung und notwendige Eingangswerte}

Die adaptierte Bilanzenmethode beruht auf der unterschiedlichen Elementarzusammensetzung von biogenen und fossilen Materialien. Während (wasserund aschefreie) biogene Materialien einen Kohlenstoff- und Sauerstoffgehalt im Bereich von $40-50 \%$ aufweisen, sind fossile Materialien (Kunststoffe) zumeist durch geringe Sauerstoffgehal- te $(<15 \%)$ und höhere Kohlenstoffgehalte $(>70 \%)$ gekennzeichnet. Dieser Unterschied wird gemeinsam mit der über Laboranalysen bestimmten Elementarzusammensetzung des Ersatzbrennstoffs verwendet, um den Anteil an biogenen bzw. fossilen Materialien zu berechnen.

Eine schematische Darstellung des Konzeptes findet sich in Abb. 2. Im Konkreten verwendet die aBM pro Element (Kohlenstoff, Wasserstoff, Stickstoff, Schwefel und Sauerstoff) eine Bilanzgleichung, wobei jede der insgesamt fünf Bilanzgleichungen die gesuchten Massenanteile an biogenen und fossilen Materialien (unbekannte Variablen $m_{B}$ und $m_{F}$ ) enthält. Die Koeffizienten und Variablen der Gleichungen werden auf wasser- und aschefreier Basis berücksichtigt. So können valide Elementarzusammensetzungen für biogene und fossile Materialien, unabhängig von ihrem Wasser- und Aschegehalt, abgeleitet und verwendet werden (Koeffizienten der linken Seite der Bilanzgleichungen). Werte der rechten Seite der Bilanzgleichungen repräsen- 


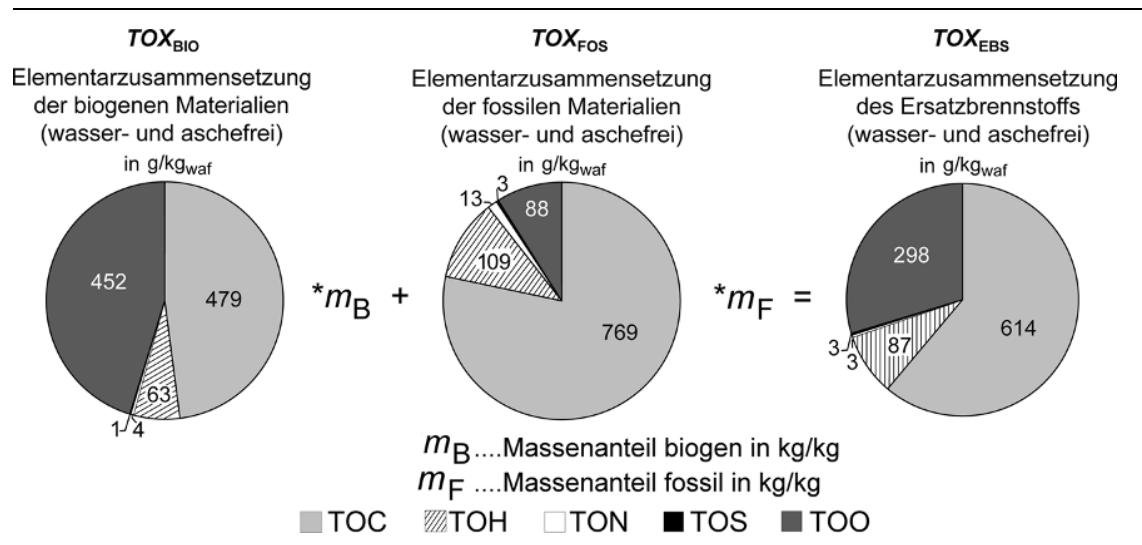

Abb. 2 Schematische Darstellung der adaptierten Bilanzenmethode anhand derer Bilanzgleichungen für TOC, TOH, TON, TOS und TOO aufgestelltwerden. (Nach Schwarzböck et al. 2016)

tieren die Elementarzusammensetzung des $\mathrm{zu}$ untersuchenden Ersatzbrennstoffs, die durch Analysen im Labor bestimmbar ist. Zusätzlich zu den fünf Bilanzgleichungen für den organischen Kohlenstoff (TOC), organischen Wasserstoff $(\mathrm{TOH})$, organischen Stickstoff (TON), organischen Schwefel (TOS), organischen Sauerstoff (TOO) gilt die Randbedingung, dass die Summe aus Massenanteil biogen und fossil per Definition gleich 1 ist. Das Gleichungssystem der aBM besteht in Summe somit aus 6 Bilanzgleichungen (TOC, TOH, TON, TOS, TOO, Summe aus $m_{\mathrm{B}}$ und $\left.m_{\mathrm{F}}\right)$ mit 2 unbekannten Massenanteilen $\left(m_{\mathrm{B}}\right.$ und $\left.m_{\mathrm{F}}\right)$. Für jeden Eingabewert des Gleichungssystems wird eine entsprechende Unsicherheit definiert. Es handelt sich somit um ein überbestimmtes System, das mithilfe nichtlinearer Ausgleichsrechnung zu lösen ist. Konkret wird dazu die Methode der schrittweisen linearen Ausgleichsrechnung angewandt. Als Ergebnis erhält man die berechneten Massenanteile biogener und fossiler Materialien $m_{\mathrm{B}}$, $m_{\mathrm{F}}$, sowie die zugehörigen Unsicherheitsbereiche (Detailinformationen in Fellner et al. 2011).

Neben dem biogenen und fossilen Massenanteil lässt sich durch die aBM auch der fossile Kohlenstoffanteil $\left(m_{F, T C}\right)$ (gleichzusetzen mit dem fossilen $\mathrm{CO}_{2}$-Anteil) ableiten. Dazu wird das Ergebnis von $m_{F}$ in die Bilanzgleichung für Kohlenstoff eingesetzt:

$$
\begin{aligned}
& m_{\mathrm{F}, \mathrm{TC}}= \\
& \left(\left(m_{\mathrm{F}} * \mathrm{TOC}_{\mathrm{FOS}}\right) *(1-A)+A * \mathrm{TIC}_{\mathrm{EBS}}\right) \quad(1) \\
& / \mathrm{TC}_{\mathrm{EBS}}
\end{aligned}
$$

mit

$m_{\mathrm{FTC}}=$ fossiler Kohlenstoffanteil in $\mathrm{kg} / \mathrm{kg}$

$m_{\mathrm{F}}=\quad$ fossiler Massenanteil in $\mathrm{kg} / \mathrm{kg}$

$T O C_{\mathrm{FOS}}=$ gesamter organischer Kohlen stoff in der fossilen Fraktion in $\mathrm{g} / \mathrm{kg}$

$A=\quad$ Aschegehalt in $\mathrm{kg} / \mathrm{kg}$

$T I C_{\mathrm{EBS}}=$ gesamter Kohlenstoff im Glührest des EBS in $\mathrm{g} / \mathrm{kg}$

$T C_{\mathrm{EBS}}=$ Gesamtkohlenstoff im EBS in $\mathrm{g} / \mathrm{kg}$

Der mittels Elementaranalysen bestimmte Kohlenstoffgehalt im EBS $\left(T C_{\mathrm{EBS}}\right)$ und in der Asche des EBS $\left(T I C_{\mathrm{EBS}}\right)$ kann hierfür verwendet werden.

Zusätzlich ermöglicht es die Methode, den biogenen und fossilen Energieanteil abzuschätzen. Dazu werden empirische Formeln zur Bestimmung des Heizwertes herangezogen, welche sich der Elementarzusammensetzung bedienen (z.B. Verbandsformel nach Dulong, Boie, Steurer oder ScheurerKestner, zitiert u.a. in Kost 2001; Liu et al. 1996; Rotter et al. 2011). In vorliegender Studie wurde die Formel von Boie nach Kost (2001) verwendet.

Schließlich können auch EBS-spezifische fossile $\mathrm{CO}_{2}$-Emissionsfaktoren berechnet werden, die die Menge an emittiertem fossilem $\mathrm{CO}_{2}$ auf die EBSMasse oder den Energieinhalt (Heizwert) beziehen:

$$
\begin{aligned}
& \text { Emissionsfaktor in } \mathrm{kg}_{\mathrm{CO} 2 \text { fos }} / t_{\mathrm{EBS}} \\
& =\mathrm{TC}_{\mathrm{EBS}} * m_{\mathrm{F}, \mathrm{TC}} * M_{\mathrm{C} 02} / M_{\mathrm{C}} \\
& \text { Emissionsfaktor in } \mathrm{kg}_{\mathrm{CO} 2 \text {,fos }} / \mathrm{GJ} \\
& =\left(\mathrm{TC}_{\mathrm{EBS}} * m_{\mathrm{F}, \mathrm{TC}} * \frac{M_{\mathrm{C} 02}}{M_{\mathrm{C}}}\right) / q_{\mathrm{EBS}}
\end{aligned}
$$
mit
$M_{\mathrm{CO} 2}$ und $M_{\mathrm{C}}=$ molare Masse von $\mathrm{CO}_{2}$ bzw. von Kohlenstoff in $\mathrm{g} / \mathrm{mol}$
$q_{\mathrm{EBS}}=\quad$ unterer Heizwert des EBS in $G J / t$

Notwendige Eingangswerte der aBM sind dementsprechend:

1. Daten zur Elementarzusammensetzung des wasser- und aschefreien Ersatzbrennstoffs $\left(T O X_{\mathrm{EBS}}\right)^{1}$. Diese sind ableitbar aus Elementaranalysen (C-, $\mathrm{H}-$, N-, S-, O-Analysen) zusammen mit der Bestimmung des Aschegehalts des Ersatzbrennstoffs.

2. Daten zur Elementarzusammensetzung der wasser- und aschefreien biogenen und fossilen Materialien im Ersatzbrennstoff (TOX $\left.\mathrm{BIO}, T O X_{\mathrm{FOS}}\right)$. Diese Informationen können der Literatur entnommen oder durch Sortierungen und anschließende Laboranalysen für den jeweiligen Ersatzbrennstoff spezifisch bestimmt werden. Eine einmalige Bestimmung der Werte für den jeweiligen Ersatzbrennstoff ist dabei in der Regel ausreichend. In vorliegender Studie wurden $T O X_{\mathrm{BIO}}$ und $T O X_{\mathrm{FOS}}$ für 6 verschiedene Ersatzbrennstoffe einzeln bestimmt, um sie mit Literaturwerten zu vergleichen und mögliche Variationen abzuschätzen (siehe Abschn. 4.2.2.).

\subsubsection{Bestimmung der EBS- spezifischen}

Elementarzusammensetzung von biogenen und fossilen Materialien

Um die Gültigkeit von Literaturwerten zur Elementarzusammensetzung biogener und fossiler Materialien (TOX und $T O X_{\mathrm{FOS}}$ aus Abb. 2) zu überprüfen sowie weitere Daten für zukünftige Anwendungen $\mathrm{zu}$ generieren, wurden detaillierte Werte zur Elementarzusammensetzung der wasser- und aschefreien biogenen und fossilen Materialien für sechs verschiedene Ersatzbrennstoffe erhoben. Diese Erhebung kann bei routinemäßiger Anwendung der aBM auf bereits bekannte Ersatzbrennstoffe entfallen. In der gegenständlichen Studie zur Validierung der aBM wurden die Ersatzbrennstoffproben manuell jeweils in 9 Fraktionen sortiert: Papier/Karton, Holz, Kunststoffe, Textilien, Organik, Gummi, Verbunde \& nicht

\footnotetext{
${ }^{1} X=\mathrm{C}, \mathrm{H}, \mathrm{N}, \mathrm{S}$ oder $\mathrm{O}$
} 
zuordenbar, Feinfraktion < 2 cm, Metalle \& inert. Für jede sortierte Fraktion wurde zuerst ein biogener bzw. fossiler Massenanteil abgeschätzt.

In einem weiteren Schritt wurden den sortierten biogenen bzw. fossilen Fraktionen die entsprechende Elementarzusammensetzung ( $\mathrm{TOC}, \mathrm{TOH}$, TON, TOS, TOO) zugeordnet. Für die Fraktionen Papier/Karton, Holz, Organik und Kunststoffe konnten dazu Analysewerte verwendet werden, da diese fast gänzlich als biogen bzw. Kunststoffe fast ausschließlich als fossil eingestuft werden können. Diese Zuordnung biogen oder fossil ist bei den Fraktionen Textilien, Gummi, Verbunde \& nicht zuordenbar sowie Feinfraktion nicht unmittelbar möglich. Folgende Annahmen und Zusatzuntersuchungen wurden daher für diese "gemischten“ Fraktionen berücksichtigt:

a) Vernachlässigung der Fraktion Gum$m i$, wenn der Massenanteil weniger als $1 \%$ ausmacht (mit Ausnahme von EBS 5, wo ein Anteil an Gummi von $1,2 \%$ gefunden wurde, konnte diese Vereinfachung angewandt werden).

b) Durchführung von Auswertungen mittels aBM unter Verwendung der Analyseergebnisse der eindeutig in biogen und fossil sortierbaren Fraktionen (Elementarzusammensetzung) für $T O X_{\mathrm{BIO}}$ und $T O X_{\mathrm{FOS}}$, um grobe Massenanteile für die gemischten Fraktionen abzuschätzen.

c) Anwendung der SLM auf einzelne Fraktionen, um biogene und fossile Massenanteile abzuschätzen.

d) Anwendung der ${ }^{14} \mathrm{C}$-Methode auf einzelne Fraktionen, um biogene und fossile Kohlenstoffanteile abzuschätzen.

e) Annahme, dass der biogene Anteil in der Fraktion Verbunde \& nicht zuordenbarPapier/Karton entspricht und der fossile Anteil aus Kunststoffen besteht, wobei angenommen wird, dass die Elementarzusammensetzung biogener und fossiler Materialien in dieser Fraktion den mittleren Werten gemäß Kost (2001) entspricht.

f) Annahme, dass die in den EBS enthaltenen Naturfasern aus $90 \%$ Zellulose und $10 \%$ Wolle bestehen und Kunstfasern aus Polyamid (30\%), Polyester $(49 \%)$, Polyacrylnitril $(15 \%)$, Polypropylen (6\%) zusammengesetzt sind (basierend auf Auswertungen in Kost 2001). Die entsprechende Elementarzusammensetzung der einzelnen Materialien folgt jeweils aus theoretischen Daten.

g) Vernachlässigung der Fraktion $\mathrm{Me}$ talle \& inert, da diese für die Auswertung des Kohlenstoffanteils nicht relevant ist.

Dadurch konnte sowohl die Sortierpräzision der „reinen“ biogenen bzw. fossilen Fraktionen abgeschätzt werden, als auch eine Annahme zur Zusammensetzung der gemischten Fraktionen getroffen werden.

Detailergebnisse zur Sortierung sowie zur Elementarzusammensetzung der einzelnen sortierten Fraktionen der untersuchten Ersatzbrennstoffe sind in Schwarzböck et al. (2017) zu finden. In selbiger Publikation konnte auch die generelle Eignung der Vorgehensweise zur Bestimmung der notwendigen Eingangsparameter $T O X_{\mathrm{BIO}}$ und $T O X_{\mathrm{FOS}}$ für die aBM bestätigt werden.

\subsection{Manuelle Sortierung (MS)}

Für die Anwendung der Manuellen Sortierung gemäß EN 15440:2011 wurden die Sortierergebnisse wie in Abschn. 4.2.2. beschrieben verwendet (Sortierung in 9 verschiedene Fraktionen). Wie für die Generierung der Eingangswerte für die $\mathrm{aBM}$ ist es auch für die MS erforderlich, einen biogenen bzw. fossilen Massenanteil pro sortierter Fraktion zuzuordnen. Für die MS wurde dafür auf Angaben in der Norm zurückgegriffen, wobei für die Fraktion Verbund keine Zuordnung vorgegeben wird und ein fossiler Massenanteil von $50 \%$ angenommen wurde. Mithilfe der sortierten Fraktionen und der jeweiligen Massenanteile in den Fraktionen kann schlussendlich ein fossiler Massenanteil pro EBS berechnet werden. Die Berechnungen dazu wurden nach EN 15440:2011 durchgeführt, wobei die Sortierpräzision gemäß Norm nicht abschätzbar war und vereinfacht mit $100 \%$ angenommen wurde. Diese Annahme scheint durch die äußerst penible Sortierung der Fraktionen gerechtfertigt. Eine Unsicherheit wurde berücksichtigt, indem auf Auswertungen einzelner sortierter Fraktionen gemäß aBM, SLM und ${ }^{14} \mathrm{C}$-Methode zurückgegriffen wurde (Ergebnisse aus Abschn. 4.2.2.).

Um von den Sortierergebnissen auch auf einen fossilen Kohlenstoffanteil zu schließen, muss ein fossiler bzw. biogener Kohlenstoffgehalt in den sortierten Fraktionen angenommen oder bestimmt werden. Hierfür wurde auf Angaben in Kost (2001) zurückgegriffen, insbesondere auf die Kohlenstoffgehalte in Papier, Holz, Wolle, gemischter Organik und gemischten Kunststoffen. Kohlenstoffgehalte in Naturfasern und Kunstfasern konnten mittels chemischer Strukturformel von Zellulose, Polyamid, Polyester, Polyacrylnitril und Polypropylen und deren jeweiliger Anteile (gemäß Kost 2001) abgeschätzt werden.

\subsection{Selektive Lösemethode (SLM)}

Die Selektive Lösemethode beruht auf der selektiven Auflösung der Probenmatrix und Oxidation der biogenen Anteile bei Behandlung mit konzentrierter Schwefelsäure und anschließender Zugabe von Wasserstoffperoxid. Die fossilen und inerten Bestandteile verbleiben - so die Erwartung - als Rückstand (EN 15440:2011).

Nicht alle Materialien folgen dieser Annahme, beispielsweise lösen sich einige biogene Substanzen nicht (z.B. Leder, Lignin, Wolle, Gummi aus Naturkautschuk) und werden somit als fossil quantifiziert, während sich insbesondere Kunststoffe mit Aminogruppen ungewollt lösen und damit fälschlicherweise als biogen eingestuft werden (z. B. Nylon, Polyurethan) (Cuperus et al. 2005; EN 15440:2011; Staber et al. 2008). Bei Vorhandensein signifikanter Anteile an den genannten Materialien führt die Methodik der SLM zu Fehleinschätzungen, wobei die Unsicherheit bzw. der mögliche Fehler zumeist schwer abzuschätzen ist.

Die SLM wurde im Zuge der Studie gemäß EN 15440:2011 angewandt. Um der Heterogenität der Proben gerecht $\mathrm{zu}$ werden, wurde eine Prüfmenge von $15 \mathrm{~g}$ gewählt (bei einer Partikelgröße von $<1 \mathrm{~mm}$ laut Norm). Zusätzlich wurden Doppelbestimmungen pro Probe durchgeführt. Der Kohlenstoffgehalt der Proben $<1 \mathrm{~mm}$, des Löserestes und des Glührestes wurde mittels katalytischer Verbrennung in einem Elementaranalysator (Elementar Vario Macro, Elementar Analysensysteme $\mathrm{GmbH}$ ) bestimmt. Über die Differenz der Kohlenstoffanalyse in der Ausgangs- und der gelösten Probe lässt sich der fossile Kohlenstoffanteil berechnen.

Gesamt wurden 25 Proben mittels SLM untersucht: 15 Papier-Rejekt-Proben, 5 EBS 1-Proben und 5 EBS 2Proben (Begrenzungen des Probenum- 
Tab. $3{ }^{14} \mathrm{C}$-Gehalt in der biogenen Fraktion der EBS, verwendet als Referenzwert zur Bestimmung des fossilen Kohlenstoffanteils mittels ${ }^{14} \mathrm{C}$-Methode

fangs aufgrund von Zeit- und Budgetlimitierung).

Die Unsicherheit der Ergebnisse wurde anhand der Doppelbestimmung (als Wiederholpräzision) und mit Verwendung von Angaben zur Vergleichspräzision in der Norm (Tabelle F. 1.4 in EN 15440:2011) berechnet.

\subsection{Radiokarbonmethode $\left({ }^{14} \mathrm{C}-\right.$ Methode $)$}

Die Radiokarbonmethode $\left({ }^{14} \mathrm{C}\right.$-Methode) stammt aus der Altersdatierung und macht sich das unterschiedliche Verhältnis von ${ }^{14} \mathrm{C}$ und ${ }^{12} \mathrm{C}$ in biogenen und fossilen Materialien zu Nutze. Der $\mathrm{F}^{14} \mathrm{C}$ ist definiert als das ${ }^{14} \mathrm{C} /{ }^{12} \mathrm{C}$ Verhältnis einer Probe im Vergleich zu dem Isotopenverhältnis des Referenzjahres 1950 (Reimer et al. 2004). Biogene Materialien haben dementsprechend einen $\mathrm{F}^{14} \mathrm{C}$ Wert von rund 1, während ${ }^{14} \mathrm{C}$ in fossilen Materialien zerfallen ist und dementsprechend der $\mathrm{F}^{14} \mathrm{C}$ Wert bei 0 liegt. Neben dem Isotopenverhältnis in der untersuchten Probe wird für die Berechnung des biogenen Kohlenstoffanteils der mittlere $\mathrm{F}^{14} \mathrm{C}$-Gehalt in der reinen biogenen Fraktion des EBS als Referenzwert benötigt. Der $\mathrm{F}^{14} \mathrm{C}$ Referenzwert ist abhängig vom Alter bzw. den Wachstumsjahren der Biomasse und bestimmt im Wesentlichen die Unsicherheit der Methode. Grund dafür sind Atomwaffentests im letzten Jahrhundert, die den ${ }^{14} \mathrm{C}$-Gehalt der Atmosphäre maßgebend verändert haben. Dennoch gilt die ${ }^{14} \mathrm{C}$-Methode unter den verfügbaren Verfahren zur Bestimmung des fossilen Anteils in Ersatzbrennstoffen als die zuverlässigste mit der geringsten analytischen Unsicherheit; z. B. Beschleuniger-Massenspektrometrie AMS $<3-7 \%$ relative Unsicherheit (Mohn et al. 2008, 2012; Palstra und Meijer 2010). Daher wird sie in dieser Studie als primäre Vergleichsmethode herangezogen.

Für die untersuchten EBS-Proben wurden Radiokarbonanalysen gemäß EN 15440:2011 mittels AMS durchgeführt. Fein zermahlene Proben wurden einer Dreifachbestimmung von je $10 \mathrm{mg}$ unterzogen. Insgesamt wurden 16 Proben analysiert: 8 Papier-Rejekt-Proben, 4 EBS 1-Proben, 4 EBS 2-Proben. Eine

\begin{tabular}{|l|l|l|l|}
\hline & Papier-Rejekt & EBS 1 & EBS 2 \\
\hline${ }^{14} \mathrm{C}$-Gehalt $\mathrm{F}^{14} \mathrm{C}_{\text {bio }}$ in pMC & $1,080 \pm 0,024$ & $1,088 \pm 0,038$ & $1,101 \pm 0,038$ \\
\hline Angaben als Mittelwert mit Standardabweichung & & \\
\hline
\end{tabular}

Bestimmung mittels ${ }^{14} \mathrm{C}$-Methode für alle Proben konnte aufgrund budgetärer Beschränkungen nicht durchgeführt werden.

Die $\quad \mathrm{F}^{14} \mathrm{C}$-Referenzwerte der verschiedenen biogenen Materialien in den EBS wurden gemäß Mohn et al. (2008) bestimmt und mithilfe der ungefähren Anteile an Papier/Karton, Holz, Textilien und Organik, die aus den Ergebnissen der manuellen Sortierung der EBS bekannt waren, kombiniert. Für Papier/Karton in Papier-Rejekt wurden zusätzliche ${ }^{14} \mathrm{C}$-Analysen durchgeführt um die Unsicherheit des Referenzwertes zu reduzieren (der Anteil von Papier/Karton in der gesamten biogenen Fraktion des Papier-Rejekts beträgt rund $92 \%$ ). $\mathrm{F}^{14} \mathrm{C}$-Referenzwerte für die biogene Fraktion in den einzelnen EBS konnten damit abgeleitet werden (Tab. 3).

Die Gesamtunsicherheit (Standardabweichung) der Ergebnisse der Radiokarbonanalysen ergibt sich vorwiegend aus der Unsicherheit des $\mathrm{F}^{14} \mathrm{C}$ Referenzwertes der enthaltenen Biomasse (wurde mit $3 \%$ relativ abgeschätzt). Hinzu kommt die Messunsicherheit aus den Messwiederholungen (2-3\%relativ).

\subsection{Chemische Analysen}

\subsubsection{Wasser- und Aschegehalt}

Der Wassergehalt der Laborproben wurde durch Trocknung bei $105^{\circ} \mathrm{C}$ für mindestens $24 \mathrm{~h}$ bestimmt (Beginn der Trocknung direkt nach der Anlieferung im Labor). Der Aschegehalt $(A)$ wurde gemäß Norm EN 15403:2011 ermittelt, indem rund $4 \mathrm{~g}$ des aufbereiteten Probenmaterials nach einer Trocknung bei $105^{\circ} \mathrm{C}$ einem Muffelofen zugeführt und bei $350^{\circ} \mathrm{C}$ für eine Stunde und anschließend unter Lufteinblasung bei $550^{\circ} \mathrm{C}$ für vier Stunden verascht wurden.

\subsubsection{Elementaranalysen}

Alle Elementaranalysen (inklusive der Kohlenstoffgehalte) wurden mittels katalytischer Verbrennung in einem Elementaranalysator (Elementar Vario Macro und Elementar Vario EL, Elementar Analysensysteme $\mathrm{GmbH}$ ) durchgeführt (gemäß DIN 51732:2014). Bei einer wurden der Gesamtkohlenstoff (TC), Gesamtwasserstoff $(T H)$, Gesamtstickstoff $(T N)$, Gesamtschwefel (TS) und Gesamtsauerstoff (TO) ermittelt, wobei die Sauerstoffbestimmung unter Sauerstoffabschluss erfolgt. Pro Probe wurden fünf Messungen mit je $40 \mathrm{mg}$ ( $T C$, $T H, T N, T S$ ) bzw. sieben Messungen mit je $4 \mathrm{mg}(T O)$ durchgeführt. Die geringere Einwaage für die $T O$-Bestimmungen ist durch eine gerätespezifische Limitierung der maximalen Sauerstoffmenge von $2 \mathrm{mg}$ absolut bedingt. samtgehalten ( $T C, T H, T N, T S, T O)$ zusammen mit dem Aschegehalt sowie Gesamtgehalten in der Asche (TIC, TIH, TIN, TIS, TIO), Elementargehalte auf wasser- und aschefreier Basis berechnet (TOC, TOH, TON, TOS, TOO) (Berechnung gemäß Schwarzböck et al. 2016, Formel (1)).

\section{Ergebnisse und Diskussion}

\subsection{Elementarzusammensetzung der biogenen und fossilen Materialien}

Für die Anwendung der aBM sind Informationen zur Elementarzusammensetzung der wasser- und aschefreien biogenen und fossilen Materialien im Ersatzbrennstoff (TOX $\left.\mathrm{BIO}, T O X_{\mathrm{FOS}}\right)$ erforderlich. In vorliegender Studie wurden diese für sechs verschiedene EBS ermittelt (siehe Abschn. 4.2.2.). Aus Abb. 3 ist ersichtlich, dass der Wertebereich für die ermittelten Daten für fast alle Parameter gut einzugrenzen ist. Insbesondere die Kennwerte für TOC und TOH liegen sowohl bei biogen als auch bei fossil für alle EBS sehr nahe beisammen und stimmen gut mit den aus der Literatur abgeleiteten Werten in Fellner et al. (2011) überein. Unter Berücksichtigung aller neu generierten Werte zeigt sich eine Variabilität für TOC und $T O H$ von weniger als $2 \%$ relativ (in biogenen Materialien) und weniger als $6 \%$ relativ (in fossilen Materialien). Am deutlichsten variieren die einzelnen Werte für TOO ${ }_{\text {FOS }}$ mit knapp $40 \%$ relativ. Diese Streuung zeugt von einer signifikanten Abhängigkeit von $T O O_{\mathrm{FOS}}$ von der KunststoffzusamVerbrennungstemperatur von $1150^{\circ} \mathrm{C}$

Für die aBM wurden aus den Ge- 


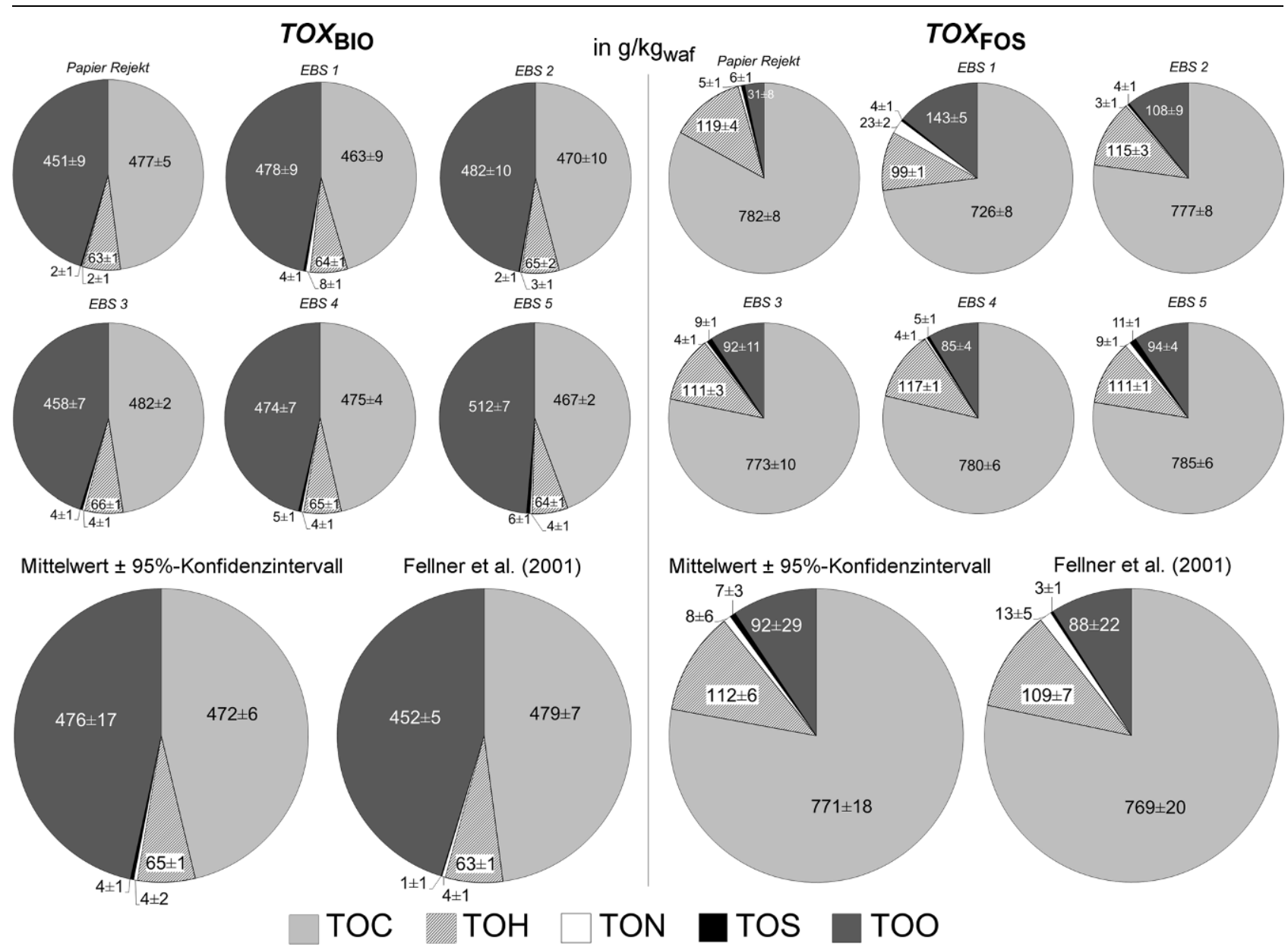

Abb. 3 Elementare Zusammensetzung der biogenen und fossilen Materialien auf wasser- und aschefreier Basis (waf), bestimmt für sechs verschiedene Ersatzbrennstoffe. Mittelwerte pro EBS mit Standardabweichung und Gesamtmittelwerte über alle EBS mit 95-\%-Konfidenzintervall

mensetzung in den EBS. Ein höherer TOO-Gehalt in den fossilen Materialien von EBS 1 (bei gleichzeitig niedrigerem TOC- und TOH-Gehalt) im Vergleich zu den anderen EBS deutet auf Anteile an Polyamid, Polyurethan oder Polyethylenterephthalat in diesem EBS hin. Diese Polymere, die einen höheren Gehalt an Sauerstoff aufweisen, dürften in den übrigen fünf EBS unwesentlich vertreten sein. In Papier-Rejekt dagegen ist anzunehmen, dass Polyethylene und Polypropylen die wesentlichen Bestandteile der Kunststofffraktion sind (TOO-Gehalt sehr gering).

Die sehr geringe Variabilität der Elementarzusammensetzung für biogene Materialien (TOX $\mathrm{BIO}$ ) weist darauf hin, dass verschiedene Anteile an Materialien in der biogenen Fraktion der EBS (z. B. Karton, Papier, Holz, Textilien) die Kennwerte nur unwesentlich beeinflussen. Trotz signifikant höherer Anteile an Papier in Papier-Rejekt
(37\%, andere EBS $<7 \%$ ) oder Textilien in EBS $1(23 \%$, andere EBS $<8 \%)$ liegen die generierten Kennwerte für diese EBS im Wertebereich der anderen EBS. Daher ist davon auszugehen, dass Daten zu TOX $\mathrm{BIO}$ auch ohne aufwendige Sortierungen und Analysen abgeleitet werden können und allgemein für die aBM einsetzbar sind.

Die generierten Kennwerte legen zudem nahe, dass typische Werte für $T O X_{\mathrm{BIO}}$ und $T O X_{\mathrm{FOS}}$ für EBS desselben Typs abgeleitet werden können. Werte aller vier untersuchten EBS, die vorwiegend aus Gewerbe- und Industrieabfällen hergestellt werden (EBS 2, EBS 3, EBS 4, EBS 5) liegen im Konfidenzintervall von $95 \%$ und zeigen unter sich sehr geringe Schwankungen bis maximal $10 \%$ relativ (für TOOFOs; exklusive TON und TOS). Es ist daher davon auszugehen, dass die generierten Kennwerte auch für andere EBS desselben Typs für die Anwendung der aBM Gültigkeit besitzen.

\subsection{Fossiler Anteil der $\mathrm{CO}_{2}$-Emissionen aus Ersatzbrennstoffen, bestimmt mittels verschiedener Methoden}

Der fossile Kohlenstoffanteil von sechs verschiedenen EBS wurde mithilfe der in Abschn. 4.2-4.5 beschriebenen Methoden bestimmt. Unter der Annahme, dass der gesamte Kohlenstoff während der Verbrennung zu CO${ }_{2}$ oxidiert, ist der fossile Kohlenstoffanteil gleichzusetzen mit dem Anteil an fossilen $\mathrm{CO}_{2}$-Emissionen nach der thermischen Verwertung der EBS.

Der Anteil der fossilen und damit klimarelevanten $\mathrm{CO}_{2}$-Emissionen für die untersuchten Ersatzbrennstoffproben wurde mit 51,8-91,1\% bestimmt (Abb. 4). Für Papier-Rejekt ist im Vergleich zu den anderen EBS ein deutlich geringerer $\mathrm{CO}_{2}$-Anteil als klimarele- 


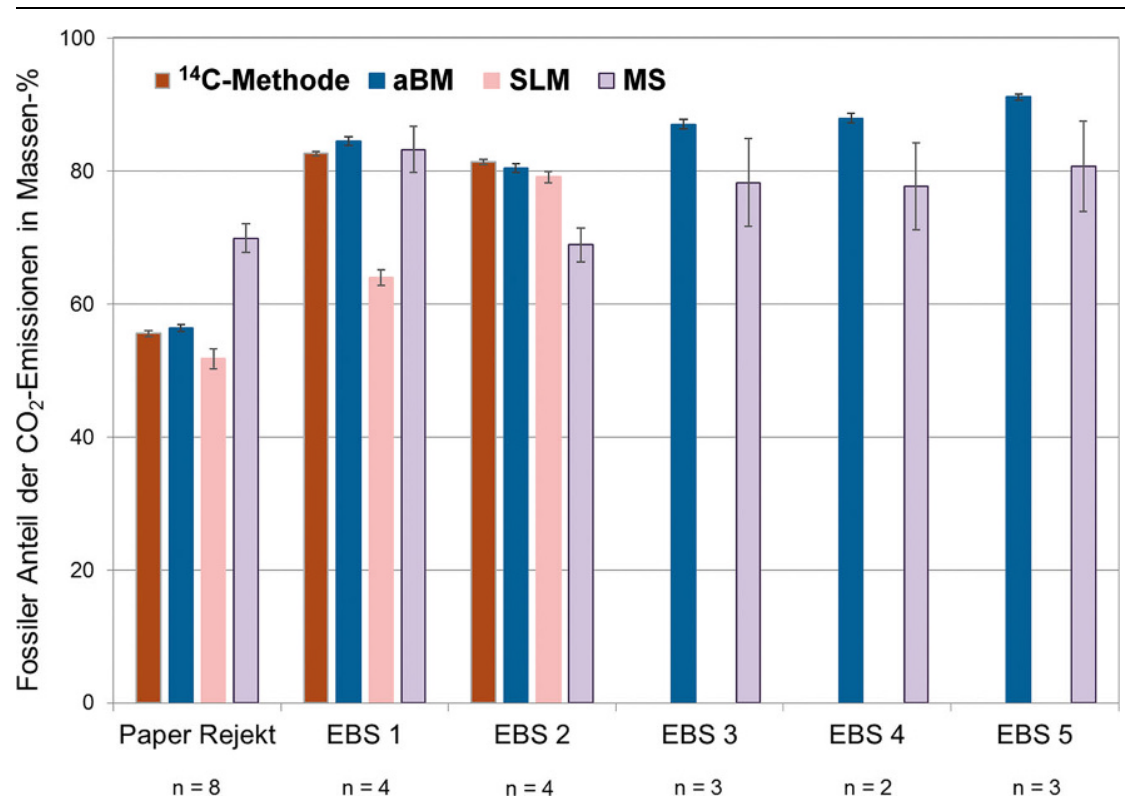

Abb. 4 Fossiler Anteil der $\mathrm{CO}_{2}$-Emissionen bestimmt für sechs verschiedene Ersatzbrennstoffe unter Anwendung unterschiedlicher Bestimmungsmethoden $\left({ }^{14} \mathrm{C}-\right.$ Methode, aBM, SLM, MS), Mittelwerte mit Standardabweichung ( $n$ Anzahl untersuchter Proben)

vant einzustufen (unter $60 \%$ ). Dies ist durch einen höheren Anteil an Papier und Karton bzw. geringeren Anteil an Kunststoffen in diesem EBS zu erklären. Laut Sortierungen ergaben sich rund $41 \%$ Kunststoffe im Papier-Rejekt, während für alle anderen EBS ein Massenanteil an Kunststoffen von über $50 \%$ gefunden wurde.

Abb. 4 zeigt eine sehr gute Übereinstimmung der mittels aBM gefundenen Werte $\mathrm{zu}$ den Werten der ${ }^{14} \mathrm{C}$-Methode (Vergleichsmessungen für 3 verschiedene EBS verfügbar). Bezogen auf den mittleren fossilen Kohlenstoffanteil dreier verschiedener EBS (PaperRejekt, EBS 1, EBS 2) liegt die mittlere Abweichung bei $0,6 \pm 1,4 \%$ absolut. Diese geringe Differenz zwischen den zwei Methoden deutet auf die Richtigkeit der aBM hin, da die ${ }^{14} \mathrm{C}$-Methode als Verfahren mit der geringsten analytischen Untersicherheit gilt. Betrachtet man die Ergebnisse der SLM im Vergleich zu den Werten der ${ }^{14} \mathrm{C}$-Methode, ist erkennbar, dass tendenziell mit einer Unterschätzung des fossilen $\mathrm{CO}_{2}$ Anteils durch die SLM zu rechnen ist. Im Konkreten weichen die Ergebnisse der SLM im Mittel $-8,2 \pm 9,1 \%$ absolut von der ${ }^{14} \mathrm{C}$-Methode ab. Der deutlichste Unterschied zwischen dem SLMErgebnis und den Werten der anderen Methoden ist für EBS 1 erkennbar, wo sich eine Unterschätzung von knapp $19 \%$ absolut im Vergleich zur ${ }^{14} \mathrm{C}$-Me- thode ergibt. Es ist anzunehmen, dass dieser höchst signifikante Unterschied auf einen vergleichsweise hohen Anteil an chemisch lösbaren Materialien fossilen Ursprungs zurückzuführen ist. In EBS 1 konnten beispielsweise relevante Massenanteile an geschäumten Kunststoffen (rund 16-26\%) und Textilien (fast $23 \%$ ) beobachtet werden; beides Materialgruppen, die potenziell Polymere mit Aminogruppen enthalten. Bei Behandlung mit konzentrierter Schwefelsäure werden diese Polymere fast gänzlich aufgelöst und deren Massenanteile daher fälschlicherweise dem biogenen Anteil zugeordnet. Diese Fehleinschätzung einzelner Materialgruppen durch die SLM ist auch in Abb. 5 erkennbar, in der fossile Kohlenstoffanteile für sortierte Fraktionen dargestellt werden. Speziell bei den Fraktionen Kunststoffe, Textilien und Verbunde zeigen sich durch die SLM Minderbefunde an fossilem Kohlenstoff.

Bei Vergleich der Ergebnisse der Manuellen Sortierung zur ${ }^{14} \mathrm{C}$-Methode sind ebenfalls signifikante Unterschiede erkennbar (Abb. 4). Auffallend ist diese Differenz vorwiegend bei Proben des Papier-Rejekts, wo im Mittel durch die MS eine Überschätzung des fossilen $\mathrm{CO}_{2}$-Anteils von 14,3 \%absolut bestimmt wurde. Bei Proben des EBS 2 hingegen ist von einer Unterschätzung durch MS auszugehen (rund $-5,4 \%$ absolut). Gesamt gesehen ergibt sich eine mittlere Abweichung der MS-Ergebnisse von ${ }^{14} \mathrm{C}$-Resultaten ohne klare Tendenz nach oben oder unten von $3,4 \pm 10 \%$ absolut. Diese Abweichungen sind auf verschiedene methodische Faktoren der MS begründet, u. a.:

a) Ein hoher Anteil der Feinfraktion (bei Papier-Rejekt mehr als 65\%) oder Textilien kann das Ergebnis verzerren, da hier gemäß EN 15440:2011 pauschal ein Massenanteil von $50 \%$ fossil angenommen wird. In den Papier-Rejekt-Proben wurde beispielsweise durch Zusatzanalysen ein fossiler Massenanteil in der Feinfraktion von nur $40 \%$ abgeschätzt, was zusammen mit dem hohen Feinfraktion anteil von $65 \%$ im Papier-Rejekt zu einer Verzerrung des gesamten fossilen Anteils von 5-6\%absolut führt. Der Textilanteil der betrachteten EBS liegt zwischen 1 und $23 \%$ (höchster Anteil in EBS 1), wobei analytisch (mittels aBM und ${ }^{14} \mathrm{C}$-Methode) ein fossiler Anteil zwischen 35 und $97 \%$ abgeschätzt wurde.

b) Eine Vorgabe für den fossilen Anteil in Verbundstoffen und nicht zuordenbaren Materialien wird in der Norm nicht gegeben. Diese Fraktion kann jedoch auch einen signifikanten Massenanteil ausmachen (z.B. in EBS 2 mehr als $40 \%$ ) und beeinflusst demnach gegebenenfalls das Ergebnis ebenfalls wesentlich.

c) Für die Abschätzung des fossilen Kohlenstoffanteils mittels MS sind Informationen zum Kohlenstoffgehalt in den sortierten Fraktionen notwendig. Durch Verwendung von Literaturwerten werden damit weitere Unsicherheiten in das Ergebnis eingebracht. Beispielsweise liegt der in dieser Studie aus der Literatur abgeleitete Kohlenstoffgehalt für biogene Materialien $T O C_{\mathrm{BIO}}$ rund $9 \%$ absolut unter dem analytisch bestimmten Wert (im Fall von Papier-Rejekt).

d) Dazu kommt klarerweise noch die Unschärfe der Massenanteile, die durch das Sortieren an sich verursacht wird und die schwer quantifizierbar ist (gemäß Norm sollte dazu SLM durchgeführt werden). Auswertungen mittels aBM, SLM, und ${ }^{14} \mathrm{C}$ Methode in der vorliegenden Studie weisen auf „Verunreinigungen“ der Papier/Karton Fraktionen und sortierten Kunststoffe von jeweils bis zu 8 Masseprozent hin. Diese Ergebnisse wurden nicht als Sortierpräzision, sondern als Unsicherheit der 


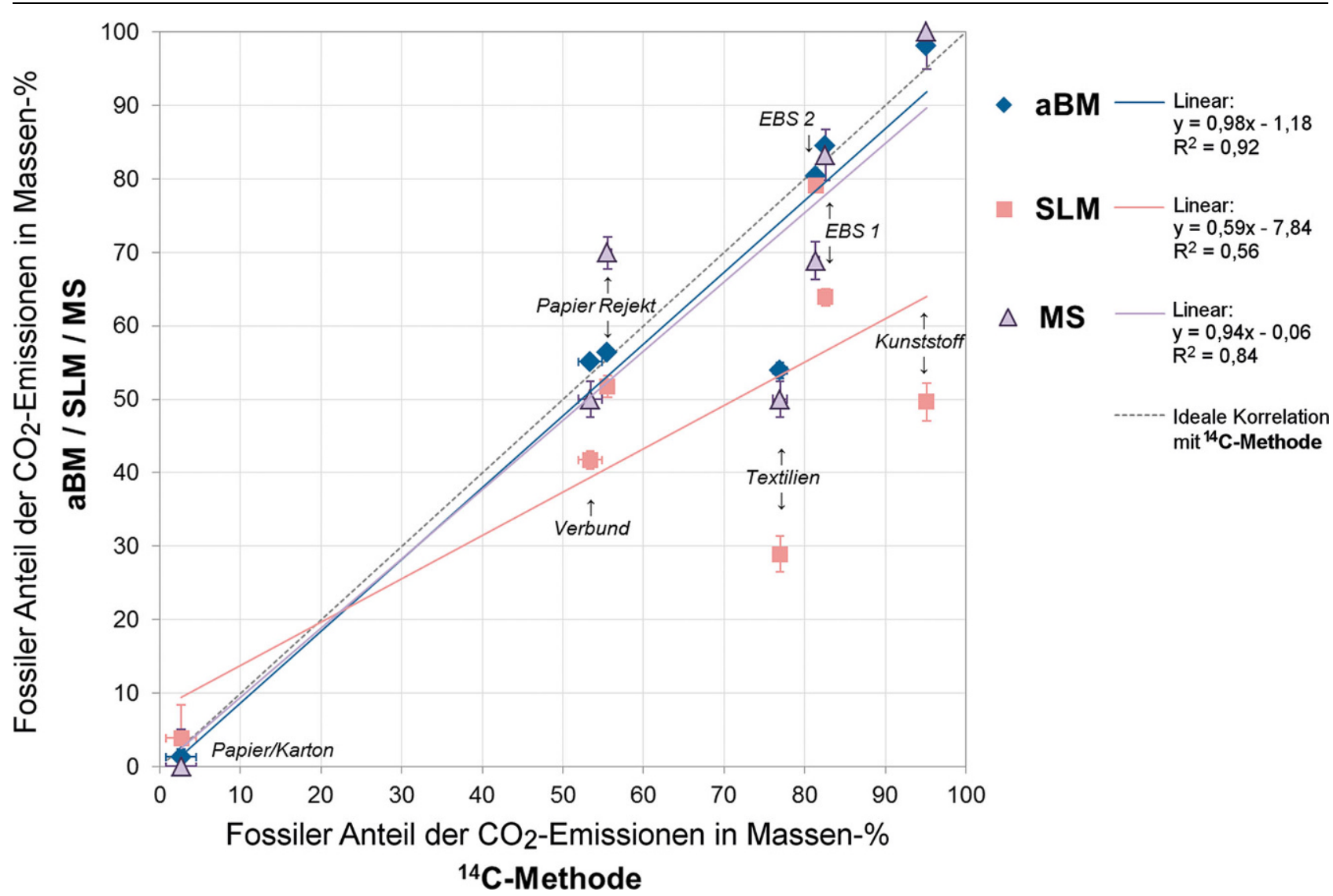

Abb. 5 Fossiler Kohlenstoffanteil bestimmt für drei Ersatzbrennstoffe und für sortierte Fraktionen der Ersatzbrennstoffe. Korrelation derErgebnisse aus ${ }^{14} \mathrm{C}$-Methode mit den Ergebnissen aus adaptierter Bilanzenemethode (aBM), Selektiver Lösemethode (SLM) und Manueller Sortierung (MS). Mittelwerte mit Standardabweichungen

MS-Ergebnisse berücksichtigt, da solche umfangreichen zusätzlichen Untersuchungen im Normalfall nicht machbar sind oder durch reine SLMAuswertungen nicht zuverlässig sind (aufgrund vorher genannter Fehleinschätzung einzelner Materialgruppen durch die SLM). Im Falle von Papier-Rejekt spielen bei der Sortierpräzision beispielsweise signifikante Anteile kleiner Partikel $(<10 \mathrm{~mm})$ oder halbaufgelöste Kartonteile und Zellulosefasern, die an Kunststoffen haften, eine Rolle.

\subsection{Fossile $\mathrm{CO}_{2}$-Emissionsfaktoren, bestimmt mittels adaptierter Bilanzenmethode}

Aus den Ergebnissen der aBM können neben den fossilen/biogenen Massenanteilen, Kohlenstoff- bzw. $\mathrm{CO}_{2}-$ Anteilen und Energieanteilen auch spezifische fossile $\mathrm{CO}_{2}$-Emissionen (fossile $\mathrm{CO}_{2}$-Emissionsfaktoren) bestimmt werden, wie sie üblicherweise für herkömmliche Energieträger angegeben werden. Damit kann ein direkter Vergleich der Brennstoffe in Bezug auf die Klimarelevanz angestellt und die gesamten fossilen $\mathrm{CO}_{2}$-Emissionen aus der thermischen Verwertung von EBS einfach bestimmt werden (z. B. für $\mathrm{CO}_{2}$ Emissionszertifikate oder die Ausweisung für die nationale Klimabilanz). Tab. 4 präsentiert die in der vorliegenden Studie bestimmten spezifischen fossilen $\mathrm{CO}_{2}$-Emissionen bezogen auf verschieden Bezugsgrößen (Feuchtsubstanz, Trockensubstanz und unterer Heizwert) für die sechs untersuchten EBS. Es ergeben sich zwischen 650 und $2190 \mathrm{~kg}$ fossiles $\mathrm{CO}_{2}$ pro Tonne EBS Feuchtsubstanz. Bei Bezug auf Trockensubstanz zeigt sich erwartungsgemäß eine etwas geringere Streuung der Werte (zwischen 1130 und $2300 \mathrm{~kg}$ fossiles $\mathrm{CO}_{2}$ pro Tonne trockener EBS). Die fossilen $\mathrm{CO}_{2}$-Emissionsfaktoren bezogen auf den Heizwert liegen zwischen 48 und $73 \mathrm{kgCO}_{2 \text {,fos }} / \mathrm{GJ}$ und damit etwas unter dem Bereich von Heizöl leicht und durchwegs unter dem Wert für
Steinkohle (jedoch meist über dem Wert für Erdgas).

Der Variationskoeffizient (relative Streuung zwischen den einzelnen untersuchten EBS-Proben) der heizwertbezogenen Emissionsfaktoren ist im Vergleich $\mathrm{zu}$ den massebezogenen Emissionsfaktoren geringer. Zur Beurteilung der Klimarelevanz und der Einsparungen an $\mathrm{CO}_{2}$ durch den Einsatz von EBS ist es förderlich, die $\mathrm{CO}_{2}$ Emissionen auf den Heizwert zu beziehen, um der tatsächlichen energetischen Substitution von fossilen Energieträgern Rechnung zu tragen. Dabei gilt es insbesondere den Wassergehalt bei der Bestimmung des Heizwertes entsprechend zu berücksichtigen. Welche Bedeutung der Wassergehalt auf den Emissionsfaktor hat, zeigt sich anschaulich für Papier-Rejekt mit einem Wassergehalt von rund $42 \%$. Der fossile $\mathrm{CO}_{2}$-Emissionsfaktor des Papier-Rejekts bezogen auf Feuchtsubstanz ist erwartungsgemäß deutlich geringer als bezogen auf Trockensubstanz. Ferner ist zu beobachten, dass sich pro Tonne feuch- 


\begin{tabular}{|c|c|c|c|c|c|c|c|}
\hline & \multirow[t]{2}{*}{$\begin{array}{l}n \\
\text { /Quelle }\end{array}$} & \multirow{2}{*}{$\begin{array}{l}\text { WG } \\
\% \text { rel }\end{array}$} & \multirow{2}{*}{$\begin{array}{l}\text { Fossiler } \mathrm{CO}_{2} \text {-Emissi- } \\
\text { onsfaktor bezogen auf } \\
\text { Feuchtsubstanz } \\
\mathrm{kgCO}_{2, \text { fos } / \mathrm{t}}\end{array}$} & \multirow{2}{*}{$\begin{array}{l}\text { VK } \\
\text { \%rel }\end{array}$} & \multirow{2}{*}{$\begin{array}{l}\text { Fossiler } \mathrm{CO}_{2-} \\
\text { Emissionsfaktor } \\
\text { bezogen auf } \\
\text { Trockensubstanz } \\
\mathrm{kgCO}_{2, \text { fos }} \text { /t was- } \\
\text { serfrei }\end{array}$} & \multirow{2}{*}{$\begin{array}{l}\text { Fossiler } \mathrm{CO}_{2} \text {-Emis- } \\
\text { sionsfaktor bezogen } \\
\text { auf unteren Heizwert } \\
\mathrm{kgCO}_{2, \text { fos }} / \mathrm{GJ}\end{array}$} & \multirow{2}{*}{$\begin{array}{l}\text { VK } \\
\text { \%rel }\end{array}$} \\
\hline & & & & & & & \\
\hline Papier-Rejekt & 15 & 42 & $650 \pm 9$ & 18 & $1130 \pm 9$ & $48 \pm 3$ & 9 \\
\hline EBS 1 & 8 & 10 & $1710 \pm 16$ & 3 & $1900 \pm 17$ & $71 \pm 3$ & 6 \\
\hline EBS 2 & 8 & 5 & $1800 \pm 16$ & 12 & $1900 \pm 17$ & $66 \pm 3$ & 6 \\
\hline EBS 3 & 3 & 11 & $1880 \pm 30$ & 3 & $2120 \pm 30$ & $70 \pm 3$ & 2 \\
\hline EBS 4 & 2 & 9 & $1840 \pm 30$ & 9 & $2030 \pm 30$ & $70 \pm 3$ & 5 \\
\hline EBS 5 & 3 & 5 & $2190 \pm 20$ & 3 & $2300 \pm 20$ & $73 \pm 3$ & 2 \\
\hline Siedlungsabfälle & IPCC $(20$ & & 557 & - & - & - & - \\
\hline Siedlungsabfälle & Anderl e & 2015) & - & - & - & 49 & - \\
\hline Industrieabfälle & Anderl e & 2015) & - & - & - & 104 & - \\
\hline $\begin{array}{l}\text { Alternativer Brennstoffmix in } \\
\text { Zementwerken }\end{array}$ & vÖZ (20 & & - & - & - & 59 & - \\
\hline $\begin{array}{l}\text { EBS (mittel- bis hochkalo- } \\
\text { risch) }\end{array}$ & Bilitews & & - & - & - & $40-54$ & - \\
\hline $\begin{array}{l}\text { EBS aus Gewerbe- und } \\
\text { Industrieabfällen }\end{array}$ & Glorius ( & 2014) & - & - & - & $50-60$ & - \\
\hline EBS aus Siedlungsabfällen & Glorius ( & & - & - & - & $20-40$ & - \\
\hline EBS aus MBA & Hoffman & I. (2010) & 470 & - & - & - & - \\
\hline $\begin{array}{l}\text { Sortierreste Leichtverpa- } \\
\text { ckungen }\end{array}$ & Hoffman & I. (2010) & 892 & - & - & 50 & - \\
\hline Erdgas & Wieser L & Irzweil (2004) & - & - & - & 55 & - \\
\hline Steinkohle & Wieser L & Irzweil (2004) & - & - & - & 95 & - \\
\hline Heizöl leicht & Wieser L & Irzweil (2004) & - & - & - & 78 & - \\
\hline \multicolumn{8}{|c|}{$\begin{array}{l}n \text { Anzahl untersuchter Proben } \\
\text { VKVariationskoeffizient: Standardabweichung über Probenergebnisse bezogen auf den Mittelwert in \%relativ } \\
\text { WG Wassergehalt bestimmt gemäß Abschn. 4.6.1, bezogen auf Feuchtsubstanz in relativen Massenprozent } \\
\text { MBA Mechanisch-biologische Anlage } \\
\text { a unterer Heizwert bestimmt mit empirischer Formel nach Boie in Kost (2001) }\end{array}$} \\
\hline
\end{tabular}

tes Papier-Rejekt fossile $\mathrm{CO}_{2}$-Emissionen ergeben, die um mindestens einen Faktor 2,6 geringer einzustufen sind als die der anderen EBS. Dieser Faktor verringert sich jedoch bei Betrachtung der fossilen $\mathrm{CO}_{2}$-Emissionen bezogen auf den Energieinhalt (Faktor von maximal 1,5). Dieses unterschiedliche Verhältnis zwischen den beiden Emissionsfaktoren ist bedingt durch einen vergleichsweise geringeren Heizwert des PapierRejekts, der durch den hohen Wassergehalt deutlich niedriger anzusetzen ist und somit die spezifischen $\mathrm{CO}_{2}$ Emissionen ansteigen lässt. Bei Berücksichtigung von $0 \%$ Wassergehalt für die Berechnung des Heizwertes läge der Emissionsfaktor für Papier-Rejekt bei nur rund $26 \mathrm{kgCO}_{2, \text { fos }} / \mathrm{GJ}$ und damit im selben Verhältnis zu den anderen EBS bei Betrachtung des massebezogenen Emissionsfaktors. Ein ähnliches Bild zeigt sich beim Vergleich von EBS 1 mit EBS 2. Der leicht höhere Kohlenstoffgehalt in EBS 2 zusammen mit einem niedrigen Wassergehalt bewirken einen höheren Heizwert und damit liegen die heizwertspezifischen fossilen $\mathrm{CO}_{2}$ Emissionen unter denen von EBS 1 (der auf die Feuchtsubstanz bezogene Emissionsfaktor von EBS 2 hingegen liegt leicht über dem von EBS 1).

Vergleicht man die generierten fossilen $\mathrm{CO}_{2}$-Emissionsfaktoren mit Angaben aus der Literatur für EBS, fällt auf, dass bisher meist geringere Werte als in vorliegender Studie berichtet werden. Dies ist natürlich in erster Linie auf andere Zusammensetzungen der jeweiligen EBS begründet, jedoch ist eine methodische Unschärfe aufgrund der in Abschn. 5.2. gefunden Differenzen der Bestimmungsmethoden nicht auszuschließen. Da die SLM in den meisten Fällen zur Anwendung kommt (relativ einfache und kostengünstige Methode), kann angenommen werden, dass es teils zu Fehleinschätzungen der fossilen $\mathrm{CO}_{2}$-Emissionsfaktoren kommt (siehe Abschn. 4.4 und 5.2).

\subsection{Praktikabilität der Bestimmungsmethoden}

Da die Wahl einer Bestimmungsmethode neben den Ansprüchen an Zuverlässigkeit auch von der Verfügbarkeit sowie dem Zeit- und Kostenaufwand abhängt, wurden die angewandten Methoden zur Bestimmung der Klimarelevanz von EBS auch auf ihre Praktikabilität hin verglichen (Tab. 5). Alle Angaben in Tab. 5 gehen von einer Bestimmung des fossilen Anteils der $\mathrm{CO}_{2}$ Emissionen für eine EBS-Probe aus. Der abgeschätzte Arbeitsaufwand für Probenaufbereitung sowie Analyse und Berechnung pro Probe reicht von rund 4-6h (SLM, aBM, ${ }^{14} \mathrm{C}-$ Methode) bis zu über $60 \mathrm{~h}$ für die MS. Bei SLM, aBM und ${ }^{14} \mathrm{C}$-Methode muss jeweils mindestens die Hälfte dieser Zeit (bei aBM sogar zwei Drittel) für die Probenaufbereitung aufgewendet werden. Dies ist durch die kleine Probengröße für die Analysen bedingt, was eine Mahlung der Proben auf sehr kleine Partikel- 
Tab. 5 Vergleich dervier Methoden bezüglichArbeits-und Zeitaufwand, Verfügbarkeitund Kosten (bezogenauf dieBestimmung des fossilen Anteils der $\mathrm{CO}_{2}$-Emissionen einer Probe)

\begin{tabular}{|c|c|c|c|c|}
\hline & Manuelle Sortierung & Selektive Lösemethode & Radiokarbonmethode & Adaptierte Bilanzenmethode \\
\hline \multicolumn{5}{|l|}{ Probenaufbereitung } \\
\hline Notwendige Arbeiten & Trocknung & $\begin{array}{l}\text { Trocknung, Mahlung, } \\
\text { Teilung, bis }<1 \mathrm{~mm}\end{array}$ & $\begin{array}{l}\text { Trocknung, Mahlung, } \\
\text { Teilung, bis }<0,2 \mathrm{~mm} \text { oder } \\
\text { feiner }\end{array}$ & $\begin{array}{l}\text { Trocknung, Mahlung, Teilung, } \\
\text { bis }<0,5 \mathrm{~mm} \text { oder feiner }\end{array}$ \\
\hline Geschätzter Aufwand & $\sim 0,5 \mathrm{~h}$ & $\sim 2 \mathrm{~h}$ & $\sim 3,5 \mathrm{~h}$ & $\sim 3,5 \mathrm{~h}$ \\
\hline $\begin{array}{l}\text { Optional (bei erstmaliger Anwen- } \\
\text { dung) }\end{array}$ & $\begin{array}{l}+\sim 18 \mathrm{~h} \\
\text { (Mahlung für C-Bestim- } \\
\text { mung der sortierten Frak- } \\
\text { tionen) }\end{array}$ & - & $\begin{array}{l}+\sim 3,5 \mathrm{~h} \\
\text { (Mahlung der sortierten } \\
\text { biogenen Fraktion) }\end{array}$ & $\begin{array}{l}+\sim 18 \mathrm{~h} \\
\text { (Mahlung für Bestimmung } \\
\text { CHNSO-Gehalt der sortierten } \\
\text { Fraktionen) }\end{array}$ \\
\hline \multicolumn{5}{|l|}{ Analyse \& Berechnung } \\
\hline Notwendige Arbeiten & Sortierung & $\begin{array}{l}\text { Bestimmung Löserück- } \\
\text { stand, Glührückstand, } \\
\text { C-Gehalt }\end{array}$ & $\begin{array}{l}\text { Graphitisierung, AMS- } \\
\text { Analyse }\end{array}$ & $\begin{array}{l}\text { Bestimmung CHNSO-Gehalt, } \\
\text { Glührückstand }\end{array}$ \\
\hline Geschätzter Analyseaufwand & $>60 \mathrm{~h}^{\mathrm{a}}$ & $\sim 1,7 \mathrm{~h}$ & $\sim 2,5 \mathrm{~h}$ & $\sim 2 \mathrm{~h}$ \\
\hline $\begin{array}{l}\text { Optional (z. B. bei erstmaliger } \\
\text { Anwendung) }\end{array}$ & $\begin{array}{l}+\sim 3-6 \mathrm{~h} \\
\text { (Bestimmung C-Gehalt } \\
\text { und Sortierpräzision der } \\
\text { sortierten Fraktionen) }\end{array}$ & - & $\begin{array}{l}+\sim 20 \mathrm{~h} \\
\text { (Sortierung und Nachmes- } \\
\text { sung des pMC Referenz- } \\
\text { wertes) }\end{array}$ & $\begin{array}{l}+\sim 25 \mathrm{~h} \\
\text { (Sortierung und Bestimmung } \\
\text { CHNS0-Gehalt der sortierten } \\
\text { Fraktionen) }\end{array}$ \\
\hline \multicolumn{5}{|l|}{ Dauer bis Analyseergebnis vorliegt } \\
\hline Geschätzte Dauer & 8 Tage & 4-5 Tage & $>20$ Tage & 3 Tage \\
\hline $\begin{array}{l}\text { Optional (z. B. bei erstmaliger } \\
\text { Anwendung) }\end{array}$ & $\begin{array}{l}+\sim 2,5 \text { Tage } \\
\text { (Bestimmung C-Gehalt der } \\
\text { sortierten Fraktionen) }\end{array}$ & - & - & $\begin{array}{l}\text { + 10 Tage } \\
\text { (Bestimmung CHNSO-Gehalt } \\
\text { der sortierten Fraktionen) }\end{array}$ \\
\hline \multicolumn{5}{|l|}{ Verfügbarkeit } \\
\hline & $\checkmark v$ & $\checkmark$ & $(\boldsymbol{\sim})$ & $\boldsymbol{V}$ \\
\hline Notwendige Laborausstattung & $\begin{array}{l}\text { Keine spezielle Ausstat- } \\
\text { tung notwendig } \\
\text { Optional: } \\
\text { Bestimmung C-Gehalt in } \\
\text { den meisten analytischen } \\
\text { Laboren verfügbar }\end{array}$ & $\begin{array}{l}\text { Bestimmung C-Gehalt in } \\
\text { den meisten analytischen } \\
\text { Laboren verfügbar; } \\
\text { für Massenanteil } \\
\text { nasschemisches Labor } \\
\text { ohne spezielle Ausstattung } \\
\text { notwendig }\end{array}$ & $\begin{array}{l}\text { Spezialisierte Geräte erfor- } \\
\text { derlich } \\
\sim 64 \text { Labore in Europa } \\
\text { ( 134 weltweit) verfügbar }\end{array}$ & $\begin{array}{l}\text { Bestimmung CHNS-Gehalt } \\
\text { in den meisten analytischen } \\
\text { Laboren verfügbar; } 0 \text {-Gehalt } \\
\text { seltener }\end{array}$ \\
\hline \multicolumn{5}{|l|}{ Analysekosten } \\
\hline $\begin{array}{l}\text { Geschätzt pro Probe (exkl. Proben- } \\
\text { aufbereitung) }\end{array}$ & $>700 €$ & $80-150 €$ & $360-650 €$ & $80-150 €$ \\
\hline \multicolumn{5}{|c|}{$\begin{array}{l}\text { âGeschätzt für eine Sortierung von } 30 \mathrm{~kg} \text { EBS und einem Arbeitsaufwand von mindestens } 2 \mathrm{~h} \text { für die Sortierung von } 1 \mathrm{~kg} \text { EBS } \\
\text { bMS: für die Ermittlung des fossilen Kohlenstoffanteils sind neben der Sortierung Informationen zum Kohlenstoffgehalt der einzelnen Fraktionen nötig, } \\
\text { diese können spezifisch bestimmt oder gegebenenfalls aus der Literatur entnommen werden; aBM: bei erstmaliger Anwendung ist es gegebenenfalls nötig } \\
\text { Sortierungen durchzuführen um EBS-spezifische Kennwerte zu generieren (Elementarzusammensetzung wasser- und aschefreier biogener und fossiler } \\
\text { Materialien). Entsprechende Datenbanken könnten hierfür diesen (in der Regel einmaligen) Zusatzaufwand deutlich reduzieren. } \\
\text { chttp://www.radiocarbon.org/Info/lablist.html (aktualisiert im Oktober 2017) }\end{array}$} \\
\hline
\end{tabular}

größen notwendig macht (bei aBM mindestens $<0,5 \mathrm{~mm}$ ). Bei der MS fließt erwartungsgemäß der überwiegende Anteil der Arbeit in die Sortierung, die jedoch stark von der ausführenden Person, der Zusammensetzung und der Struktur des EBS abhängig ist. Neben dem Arbeitsaufwand wird die Dauer, bis ein Analysenergebnis vorliegt, $\mathrm{zu}$ einem wesentlichen Anteil durch Warte- und Reaktionszeiten bestimmt (bei SLM, ${ }^{14}$ C-Methode und aBM). Diese Dauer wird bei Anwendung der aBM mit rund 3 Tagen abgeschätzt, bei SLM mit 4-5 Tagen, bei MS mit rund 8 Tagen und mit mehr als 20 Tagen bei Anwendung der ${ }^{14} \mathrm{C}$-Methode. Die bestimmenden Faktoren sind dabei die Dauer der Sortierung (MS), Dauer der CHNSOund Aschegehaltsbestimmungen (aBM, SLM), die Löse- und Oxidationsreaktionen (SLM) und die Überführung zu Graphit $\left({ }^{14} \mathrm{C}\right.$-Methode). Bei erstmaliger Anwendung oder bei sehr hohen Ansprüchen an die Genauigkeit der Methoden können zudem Zusatzanalysen notwendig sein. Um die Richtigkeit zu erhöhen, macht es möglicherweise
Sinn, die sortierten Fraktionen der MS auf ihren Kohlenstoffgehalt zu untersuchen, statt auf Literaturwerte zurückzugreifen. Für die ${ }^{14} \mathrm{C}$-Methode kann die Nachmessung des pMC-Referenzwertes des reinen biogenen Materials im EBS die Unsicherheit der Methode reduzieren, was ebenfalls zusätzliche Analytik erfordert. Wenn notwendige Informationen für die aBM zur Elementarzusammensetzung der wasserund aschefreien biogenen und fossilen Materialien nicht aus der Literatur ableitbar oder aus einer Datenbank ent- 
nommen werden können, dann sind einmalig Sortierungen und Elementaranalysen der sortierten Fraktionen erforderlich (beispielsweise wie in Abschn. 4.2.2 beschrieben).

Weitere wesentliche Faktoren für die Praktikabilität einer Methode sind zudem die Verfügbarkeit und die Analysekosten. Beispielsweise kann die ${ }^{14} \mathrm{C}$ Methode nur von Labors mit hoch spezialisierter Ausstattung durchgeführt werden, während die notwendigen Analysegeräte für die Bestimmung mittels aBM und SLM in den meisten analytischen Labors zur Verfügung stehen. Sollte ausschließlich der fossile bzw. biogene Massenanteil gefragt sein, kann die SLM in jedem nasschemischen Labor angewandt werden. Ebenso ist für die MS außer zum Trocknen und Wiegen der Proben keine spezielle Laborausstattung notwendig.

Die Komplexität und Verfügbarkeit der Analysen spiegelt sich in den Analysekosten, insbesondere bei denen der ${ }^{14} \mathrm{C}$-Methode wider. Die ungefähren Kosten liegen mit 360-650€ pro Messung im Vergleich $\mathrm{zu}$ aBM und SLM um den Faktor 2-8 höher. Die Kosten für eine Bestimmung des fossilen Kohlenstoffanteils mittels aBM und SLM liegen für beide Methoden im Bereich zwischen 80 und $150 €$ pro Probe.

\section{Schlussfolgerungen}

Die vorliegende Studie zeigt, dass die Methodik der adaptierten Bilanzenmethode (aBM) als Alternative zu standardisierten Verfahren zur Bestimmung des fossilen Kohlenstoffanteils in EBS angewandt werden kann. Es ist eine sehr gute Übereinstimmung zwischen generierten Werten der aBM und Ergebnissen der Vergleichsmethode $\left({ }^{14} \mathrm{C}\right.$ Methode) $\mathrm{zu}$ verzeichnen. Eine mittlere Abweichung von der ${ }^{14} \mathrm{C}$-Methode von $0,6 \pm 1,4 \%$ absolut bezogen auf den mittleren Anteil an fossilen $\mathrm{CO}_{2}$-Emissionen drei verschiedener EBS deutet auf die Richtigkeit der aBM-Ergebnisse hin. Für die zwei weiteren angewandten und bereits standardisierten Methoden - die Selektive Lösemethode (SLM) und die Manuelle Sortierung (MS) zeigen sich deutliche methodische Einschränkungen in Abhängigkeit von der Zusammensetzung der EBS. Die aBM ist damit neben der ${ }^{14} \mathrm{C}$-Methode die einzige Methode mit der, unabhängig vom EBS-Typ, zuverlässige Werte zur Klimarelevanz generiert werden konnten.
Die Anwendung der SLM führte tendenziell zu einer Unterschätzung des fossilen Kohlenstoffanteils, wobei diese Fehleinschätzung besonders deutlich bei Anwendung auf EBS 1 auftritt (knapp 19\% absolute Abweichung von der ${ }^{14} \mathrm{C}$-Methode). Dieser EBS wird unter anderem aus aufbereiteten Siedlungsabfällen hergestellt und enthält signifikante Anteile an fossilen Bestandteilen, die sich bei chemischer Behandlung auflösen und damit mittels SLM fälschlicherweise als biogen eingestuft werden.

Die Zuverlässigkeit der MS-Ergebnisse wird neben den unbekannten Unsicherheiten aus der manuellen Sortierung, auch durch signifikante Anteile an nicht sortierbaren Fraktionen mit unbekannten Anteilen an biogenen oder fossilen Materialien beeinflusst (Feinfraktion, Textilien, Verbunde, Gummi).

Kritischster Faktor bei der Anwendung der aBM ist die Wahl der Eingangswerte zur Elementarzusammensetzung der wasser- und aschefreien biogenen und fossilen Materialien im Ersatzbrennstoff ( $T O X_{\mathrm{BIO}}, T_{\mathrm{FOS}}$ ). Diese Werte werden idealerweise für jeden EBS spezifisch erhoben (durch einmalige Sortierungen und Analysen). Die Ergebnisse der vorliegenden Studie zeigen jedoch, dass die Werte, generiert für 6 verschiedene EBS, in einem sehr engen Bereich liegen. Es kann also angenommen werden, dass zukünftig Arbeitsaufwand für die Erstellung dieser Kennwerte eingespart werden kann, indem auf vorhandene Literaturwerte zurückgegriffen wird. Da jedoch Variationen in der Zusammensetzung der Kunststoffe in den verschiedenen EBS nicht ausgeschlossen werden können (erkennbar hauptsächlich an den unterschiedlichen $T_{O} O_{\text {FOS }}-$ Gehalten), scheint es sinnvoll, eine Datenbasis mit typischen Kennwerten zur Elementarzusammensetzung biogener und fossiler Materialien pro EBS-Typ zu erstellen. Beispielsweise enthalten EBS aus Siedlungsabfällen typischerweise höherer Anteile an Kunststoffen mit hohem TOO-Gehalt (z.B. Polyamid, Polyethylenterephthalat, Polyurethane) als EBS produziert aus Gewerbe- und Industrieabfällen (vorwiegend Polyethylen und Polypropylen).

Die aBM kann neben ihrer Zuverlässigkeit auch mit ihrer Praktikabilität gegenüber standardisierten Bestimmungsmethoden punkten. Einzig bei erstmaliger Anwendung auf einen EBS kann eine Ermittlung von EBS-spezi- fischen Eingangswerten den Kostenund Zeitaufwand etwas erhöhen. In einer Routineanwendung übertrifft sie jedenfalls die momentan vorwiegend angewandte SLM in ihrer Zweckmäßigkeit, da Analysewerte verlässlicher und meist schneller generiert werden können. Im Vergleich zur ${ }^{14} \mathrm{C}$-Methode, die sehr zuverlässige Werte liefert, ist die aBM deutlich kostengünstiger und kann von einer wesentlich größeren Anzahl an Labors durchgeführt werden. Die Methode der MS kann für eine Routinebestimmung des fossilen $\mathrm{CO}_{2}$-Anteils als nur bedingt zweckmäßig eingestuft werden, da sie neben den unbekannten Unsicherheiten auch einen sehr hohen Arbeitsaufwand erfordert.

Zusammenfassend lässt sich festhalten, dass die aBM methodische und monetäre Einschränkungen standardisierter Methoden überwindet und gleichzeitig eine praktikable und zuverlässige Anwendung erlaubt.

Danksagung Vielen Dank an die Betreiber der Ersatzbrennstoff- und Altpapieraufbereitungsanlagen für die Unterstützung und Mitwirkung bei den Probenahmen. Zusätzlicher Dank gilt Edith Vogel für die Unterstützung bei ${ }^{14} \mathrm{C}$-Analysen, dem Team des Labors der TU Wien und Inge Hengl für die Unterstützung bei der grafischen Gestaltung.

Förderung Die Autoren danken dem Fonds zur Förderung der wissenschaftlichen Forschung (FWF), Projektnummer TRP 285-N28, für die Finanzierung der Studie sowie dem Bundesministerium für Wissenschaft, Forschung und Wirtschaft für die finanzielle Unterstützung.

Funding Open access funding provided by TU Wien (TUW).

Open Access Dieser Artikel wird unter der Creative Commons Namensnennung 4.0 International Lizenz (http:// creativecommons.org/licenses/by/4. 0/deed.de) veröffentlicht, welche die Nutzung, Vervielfältigung, Bearbeitung, Verbreitung und Wiedergabe in jeglichem Medium und Format erlaubt, sofern Sie den/die ursprünglichen $\mathrm{Au}$ tor(en) und die Quelle ordnungsgemäß nennen, einen Link zur Creative Commons Lizenz beifügen und angeben, ob Änderungen vorgenommen wurden. 
Anderl, M., Haider, S., Lampert, C., Moosmann, L., Pazdernik, K., Pinteris, M., Poupa, S., Purzner, M., Schmid, C., Schmidt, G., Schodl, B. Stranner, G., Schwaiger, E., Schwarzl, B., Weiss, P., Wieser, M., Zechmeister, A. (2015): Austria's national inventory report 2015-Submission under the United Nations Framework Convention on Climate Change. Umweltbundesamt GmbH (Hrsg) REP-0552. ISBN: 978-3-99004364-6. Vienna, Austria, $585 \mathrm{~S}$

Aschenbrenner, P., Fellner, J., Rechberger, $\mathbf{H}$ (2009): Bestimmung des biogenen Kohlenstoffgehaltes von Ersatzbrennstoffen mittels eines CHNSO-Elementaranalysators. In: Thomé-Kozmiensky KJ, Beckmann M (Hrsg) Erneuerbare Energien, Bd Band 2. Vivis Verlag, Neuruppin, Deutschland, S 3-14

Bilitewski, B. (2006): Berechnung von $\mathrm{CO}_{2}$-Vermeidungskosten für die Optimierung von Abfallverbrennungsanlagen. In: Urban AI, Bilitewski B, Faulstich M (Hrsg) 11. Fachtagung Thermische Abfallbehandlung, München, Deutschland 2006. Schriftenreihe des Fachgebietes Abfalltechnik, Universität Kassel, S 187-196

cemsuisse (2016): Jahresbericht des cemsuisse (Verband der Schweizerischen Cementindustrie), http://www.schweizer-zement.ch, Bern Schweiz, $19 \mathrm{~S}$

Cuperus, J.G., van Dijk, E.A., de Boer, R.C (2005): Pre-normative research on SRF. TAUW bv, European Recovered Fuel Organisation (ERFO), Deventer, Netherlands, $128 \mathrm{~S}$

DIN 51732:2014: Prüfung fester Brennstoffe Bestimmung des Gesamtgehaltes an Kohlenstoff, Wasserstoff und Stickstoff - Instrumentelle Methoden. Deutsches Institut für Normung e.V., Berlin, $10 \mathrm{~S}$

EN 15403:2011: Feste Sekundärbrennstoffe Verfahren zur Bestimmung des Aschegehalts. Deutsches Institut für Normung e. V., Berlin, $14 \mathrm{~S}$ EN 15440:2011: Feste Sekundärbrennstoffe Verfahren zur Bestimmung des Gehaltes an Biomasse. Deutsches Institut für Normung e.V., Berlin, $60 \mathrm{~S}$

Fellner, J., Aschenbrenner, P., Cencic, O., Rechberger, H. (2011): Determination of the biogenic and fossil organic matter content of refusederived fuels based on elementary analyses. Fuel 90 (11):3164-3171. doi: https://doi.org/10.1016/ j.fuel.2011.06.043

Glorius, T. (2006): Erfahrungen mit Produktion und Einsatz gütegesicherter Sekundärbrennstoffe - RECOFUEL. In: Urban AI, Bilitewski B, Faulstich M (Hrsg) 11. Fachtagung Thermische Abfallbehandlung, München, Deutschland,
2006. Schriftenreihe des Fachgebietes Abfalltechnik, Universität Kassel, S 289-308

Glorius, T. (2014): Production and use of Solid Recovered Fuels-developments and prospects, REMONDIS GmbH, Köln. ZKG 9 (2014), Special Issue AFR, Bauverlag, Gütersloh, S 72-80 IPCC (2000): IPCC Good Practice Guidance and Uncertainty Management in National Greenhouse Gas Inventories-Chapter 5-Waste. http://www.ipcc-nggip.iges.or.jp/public/gp/ english/, $32 \mathrm{~S}$

ISO 18466:2016: Stationary source emissions Determination of the biogenic fraction in $\mathrm{CO}_{2}$ in stacks gas using the balance method. International Organization for Standardization (ISO), https://www.iso.org/standard/62513.html. 25 S Kost, T. (2001): Brennstofftechnische Charakterisierung von Haushaltsabfällen (Dissertation). Beiträge zur Abfallwirtschaft/Altlasten, Bd 16 Schriftenreihe des Instituts für Abfallwirtschaft und Altlasten, Technische Universität Dresden Deutschland, $102 \mathrm{~S}$

Liu, J.-I., Paode, R.D., Holsen, T.M. (1996): Modeling the Energy Content of Municipal Solid Waste Using Multiple Regression Analysis. J Air Waste Manage 46 (7):650-656. https://doi.org/ 10.1080/10473289.1996.10467499

Mohn, J., Szidat, S., Fellner, J., Rechberger, H. Quartier, R., Buchmann, B., Emmenegger, L. (2008): Determination of biogenic and fossil $\mathrm{CO} 2$ emitted by waste incineration based on ${ }^{14} \mathrm{CO}_{2}$ and mass balances. Bioresource Technol 99 (14):6471-6479. doi: https://doi.org/10.1016/ j.biortech.2007.11.042

Mohn, J., Szidat, S., Zeyer, K., Emmenegger, L. (2012): Fossil and biogenic $\mathrm{CO}_{2}$ from waste incineration based on a yearlong radiocarbon study. Waste Manage 32 (8):1516-1520. doi: https://doi. org/10.1016/j.wasman.2012.04.002

Palstra, S.W.L., Meijer, H.A.J. (2010): Carbon-14 based determination of the biogenic fraction of industrial $\mathrm{CO}_{2}$ emissions-Application and validation. Bioresource Technol 101 (10):3702-3710. doi: https://doi.org/10.1016/j.biortech.2009.12. 004

Rahman, A., Rasul, M.G., Khan, M.M.K., Sharma, S. (2015): Recent development on the use of alternative fuels in cement manufacturing process. Fuel 145:84-99. doi: https://doi.org/10. 1016/j.fuel.2014.12.029

Reimer, P.J., Brown, T.A., Reimer, R.W. (2004): Discussion: Reporting and Calibration of PostBomb ${ }^{14} \mathrm{C}$ Data. Radiocarbon 46 (3):1299-1304. doi: https://doi.org/10.1017/S0033822200033154
Rotter, V.S., Lehmann, A., Marzi, T., Möhle, E., Schingnitz, D., Hoffmann, G. (2011): New techniques for the characterization of refuse-derived fuels and solid recovered fuels. Waste Manage Res 29:229-236. doi: https://doi.org/10.1177/ $0734242 X 10364210$

Schwarzböck, T., Aschenbrenner, P., Rechberger, H., Brandstätter, C., Fellner, J. (2016): Effects of sample preparation on the accuracy of biomass content determination for refuse derived fuels. Fuel Process Technol 153:101-110. doi: https://doi.org/10.1016/j.fuproc.2016.07.001

Schwarzböck, T., Aschenbrenner, P., Mühlbacher, S., Szidat, S., Spacek, S., Fellner, J. (2017) Determination of the climate relevance of refuse derived fuels-Validity of literature-derived values in comparison to analysis-derived values. In: Cossu R, Stegmann R (Hrsg) CDProceedings Sardinia 2017, 16th International Waste Management and Landfill Symposium "Sardinia Symposium 30th Anniversary Book“, 2.10.-06.10.2017, Santa Margherita die Pula, Sardinia, Paper No. 236. CISA Publisher, Padova, Italy, S 1-20

Staber, W., Flamme, S., Fellner, J. (2008): Methods for determining the biomass content of waste. Waste Manage Res 26 (1):78-87. doi: https:// doi.org/10.1177/0734242x07087313

Hoffmann, G., Wünsch, C., Brunn, L., Schnapke, A., Schingnitz, D., Günther, M., Baumann, J., Wagner, J., Bilitewski, B. (2010): Nutzung de Potenziale des biogenen Anteils im Abfall zur Energieerzeugung. Umweltbundesamt (Hrsg). Institut für Abfallwirtschaft und Altlasten der Technischen Universität Dresden und INTECUS $\mathrm{GmbH}$, Pirna, Deutschland, $284 \mathrm{~S}$

VDZ (2017): Struktur des Energieeinsatzes in der Zementindustrie in Deutschland nach Energieträger im Jahr 2015. Verein Deutscher Zementwerke (VDZ). https://de.statista.com/statistik/ daten/studie/240738/umfrage/brennstoffmixder-zementindustrie-in-deutschland/. Zugegriffen: 2017-08-01

VÖZ (2015): Nachhaltigkeitsbericht 2014 der österreichischen Zementindustrie. Verein der österreichischen Zementindustrie (VÖZ), Wien, Österreich, $19 \mathrm{~S}$

Wieser, M., Kurzweil, A. (2004): Emissionsfaktoren als Grundlage für die österreichische Luftschadstoff-Inventur (Stand 2003). Umweltbundesamt $\mathrm{GmbH}$ (Hrsg) Bericht BE-254. ISBN 3-85457-749-4. Wien, Österreich, 23 S 